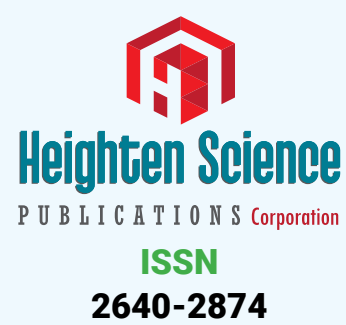

2640-2874
*Address for Correspondence: Mauro Luisetto, Applied Pharmacologist, European Specialist Lab Medicine, Independent Researcher, Italy, Email: maurolu65@gmail.com

Submitted: 28 November 2018

Approved: 13 December 2018

Published: 14 December 2018

Copyright: ๑ 2018 Luisetto M, et al. This is an open access article distributed under the Creative Commons Attribution License, which permits unrestricted use, distribution, and reproduction in any medium, provided the original work is properly cited

Keywords: Lower urinary tract disease Pharmacology; Receptors, Physio-pathology; $\mathrm{BPH}$; Emorragic cystitis

Check for updates
Research Article

\section{Receptor pharmacology and other relevant factors in lower urinary tract pathology under a functional and toxicological approach: Instrument to better manage antimicrobials therapy}

\author{
Mauro Luisetto1*, Naseer Almukhtar², Behzad Nili- \\ Ahmadabadi ${ }^{3}$, Ghulam Rasool Mashori ${ }^{4}$, Kausar Rehman \\ Khan ${ }^{5}$, Ram Kumar Sahu ${ }^{6}$, Farhan Ahmad Khan7, Gamal Abdul \\ Hamid $^{8}$ and Luca Cabianca ${ }^{9}$ \\ 'Applied Pharmacologist, European Specialist Lab Medicine, Independent Researcher, Italy \\ 2Professor, Physiology, College of Medicine, University of Babylon, Hilla, Iraq \\ ${ }^{3}$ PharmD, Nano Drug Delivery (a product development firm), Chapel Hill NC, USA \\ 4Professor of Pharmacology, People University of Medical \& Health Sciences for Women, \\ Nawabshah, Pakistan \\ ${ }^{5}$ Preston University, Karachi, Pakistan \\ ${ }^{6}$ Associate Professor, Deendayal Upadhyay Memorial Health Science and Ayush University of \\ Chhattisgarh, Raipur, India \\ ${ }^{7}$ Associate Professor and Head Department of Pharmacology Government Medical College \\ Shahdol, M.P, India \\ ${ }^{8}$ Professor, Hematology Oncology, University of Aden, Aden, Yemen \\ ${ }^{9}$ Biomedical Laboratory, Italy
}

\section{Abstract}

In various patients conditions involved in lower urinary tract disease LUT (like overactive bladder, bladder neck sclerosis, dis - synergy (with our synenrgic contraction between bladder detrusor and bladder neck, BPH, recurrent cysytitis, interstitial cystitis, chronic prostatitis, uretral stenosys, loss of sfinteric coordination.

Prostatic cancer, anatomic abnormalities and other the receptor status play relevant role to reduce effect of vicious clycle that can be responsible in progression of the pathologic process.

In this work the complex receptorial status is analyzed to verify new therapeutic strategies.

Starting from the observation that various irritant substanties produce irritant stimulus in Prostatic Patients or in bladder neck condition is interesting to deep understand the etiopatogenesys and Functional results.

In Various prostatic, bladder neck or ureteral condition a reduced urinary fluss can produce infectious.

Conditions like acute or chronic prostatitis.

Irritants sustanties in diet (in example etilic alcohol drink, hot spices, crud meats, carbonate drinks, caffeine and other) can produce Painful stimulus in innervations of vecical trigonous, bladder neck and prostatic urethra.

The same recurrent cystitis and Bph contribute in a complex situation.

This stimulus produce ipertonus of bladder muscle involved in the expulsion of urine. 
The event related inflamation and edema (bladder, prostatic uretra, trigonus) contribute to the global effect.

So conditions like bladder neck sclerosys IPB, recurrent prostatitis and cistitys in acts in a vicious circle. (Also immunomediated: Bph and cronic prostatitis with linfocite infiltration and tissue remodeling).

The ormonal status check the systems (see 5-ARI efficacy in Bph).

Simpatic, parasimpatic and other system are deeply involved.

Also behavioral habits or diet can influence in example urinary flux in a complex system like LUT. (Bladder and prostatic irritants that can produce edema and acute inflamation).

Other behavior habits are deeply involved as too much sedentary, water intake, coffee, pee modality and also psychological profile and stressing conditions.

Some disease like diabetes produce high consequences in all this systems due to

Bladder modification, oxidative stress, osmotic movens, and increase susceptibility of urinary infections.

This article are verified this kind of movens that contribute in physio -pathology of some low urinary tract conditions.

The anatomic abnormalities produces, obviously, physiological disfuntions.

Recurrent urinary tract infections, inadequate antimicrobial therapy:

Profile of resistance, duration of therapy, kind of antimicrobials, posology,

Pk. Kinetics, associations, compliance, biofilms, micro calcifications (recurrent chronic prostatitis) contribute to a progression of the condition

\section{Introduction}

This work is produced under a pharmaceutical- pharmacological, pathophysiological and toxicological point of view and it focused on male individuals and not under a surgery light.

Various receptors control the LUT like cholinergic, alfa drenergic, nociceptors, PDE5, androgen and other are involved in physio-pathology of this conditions (Figures 1-13).

Colinergic, adrenergic innervation in bladder and bladder neck, adrenergic in prostate, androgen receptor, PDE5 rec., nociceptors but also other system contribute in the global Physiology and pathology.

Various classic drugs and remedies are used towards this systems and mediator like: anticholinergics, selective alfa blokers, 5- ARI, FANS, cortisons, antidemigen, phyto-terapy, botulism toxins, antimicrobials, phyto-therapic and other.

(But according biomedical literature other system are involved)

http://www.prostatite.info/upoints.html

Muscle involved in bladder voiding:

Bladder detrusor: smooth muscle

Urethra: smooth and striated muscle

Pelvic floor: striated muscle

Pelvic nerve parasimpatic (ach)

epigasttric nerve sympathetic (NA) b3, alfa 1 bladder neck and posterior urethra

Pudend nerve: somatomotor ach

Prostate: alfa 1 rec, androgen receptors, PDE5 


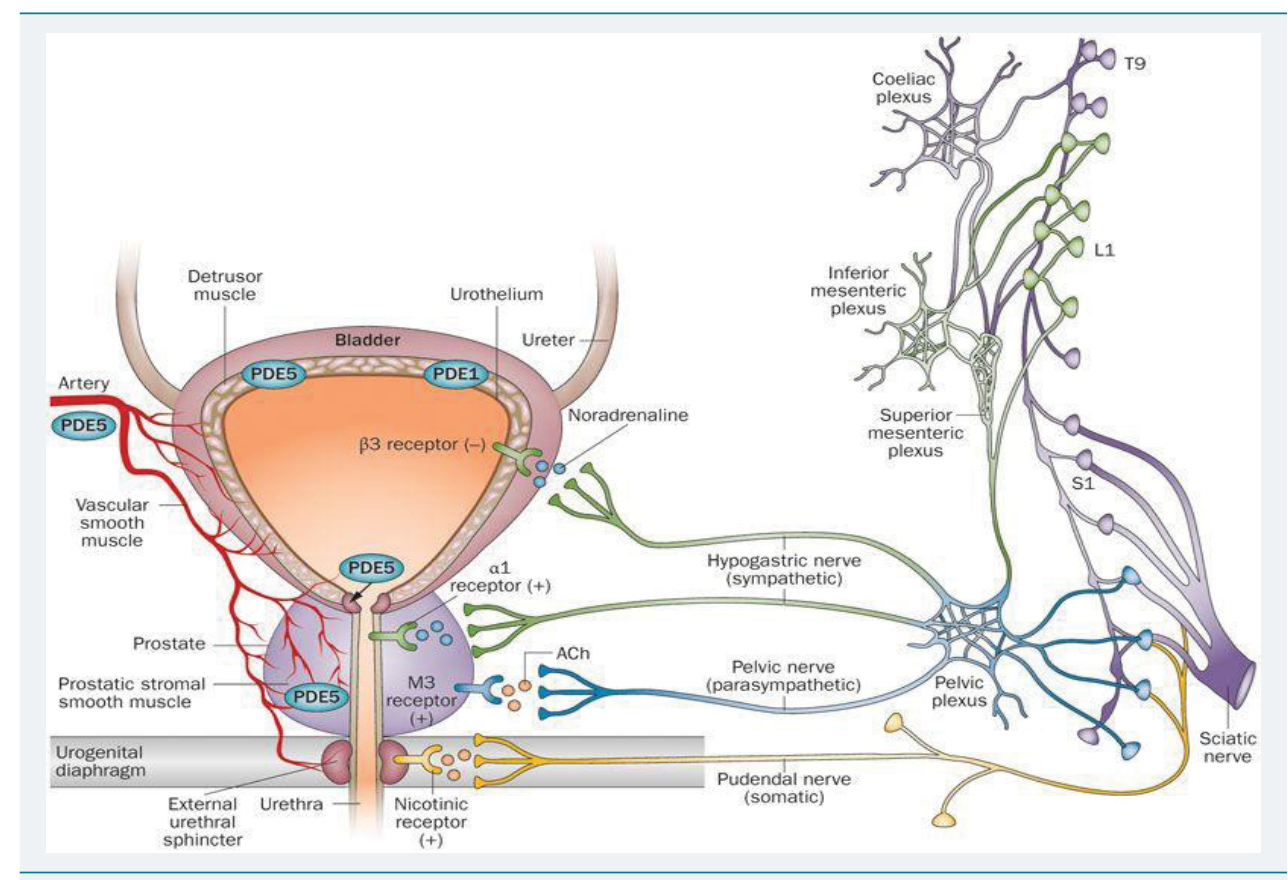

Figure 1: Innervation and receptor in men LUT; Lower urinary tract innervation and receptors.
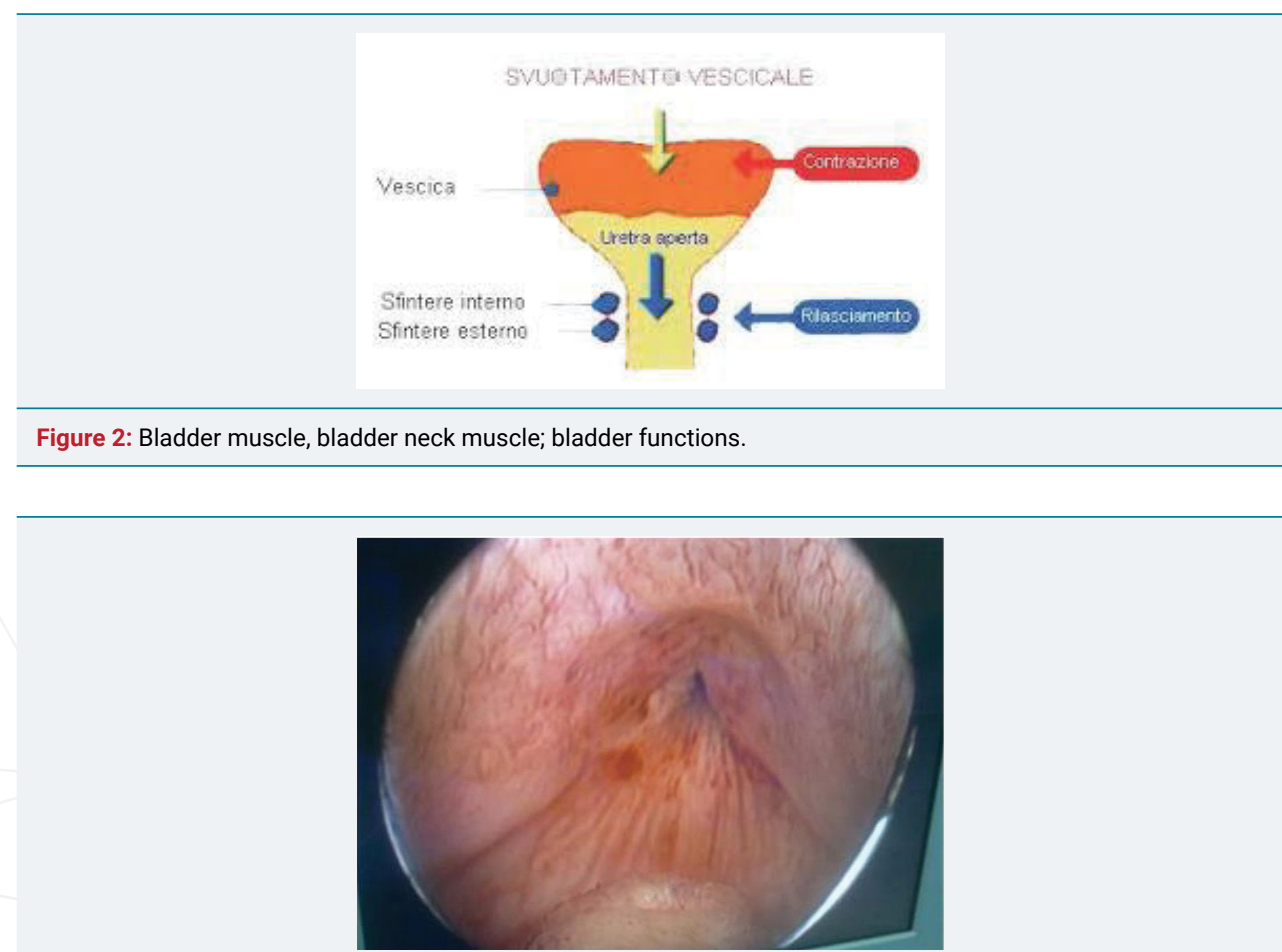

Figure 3: Bladder neck sclerosis.
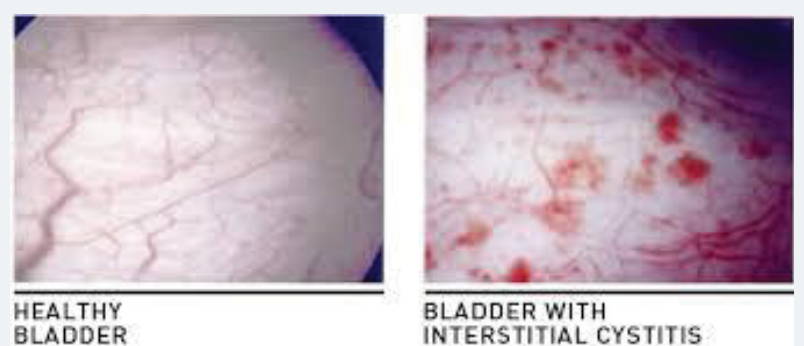

Figure 4: Show healthy bladder and bladder with cystitis 


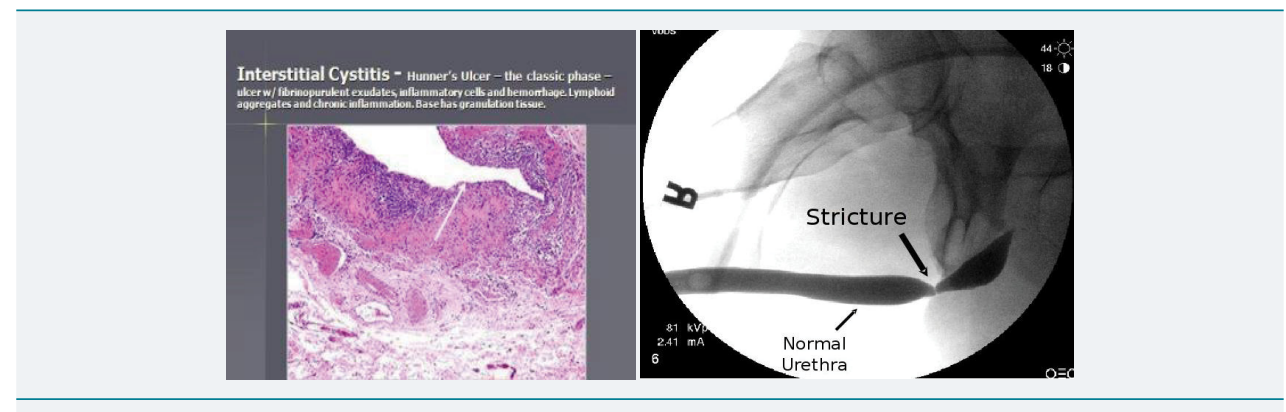

Figure 5: Show the hysto-patological changes in interstitial bladder cystitis. urehral stenosis.

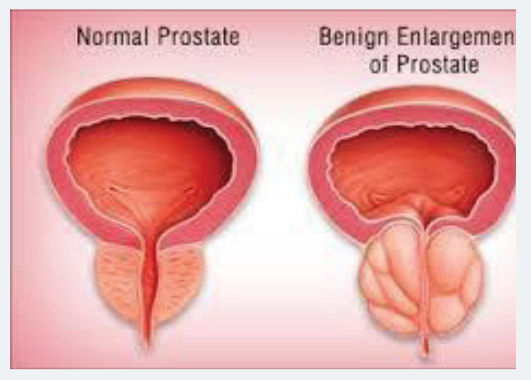

Figure 6: BPH show the benign prostatic hyperplasia.

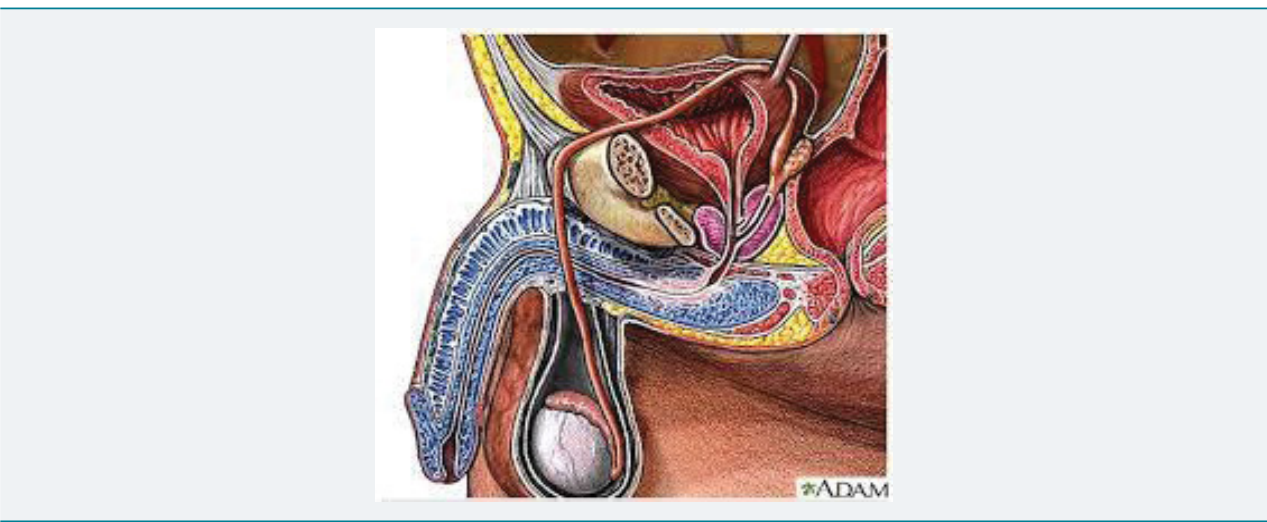

Figure 7: Posterior urethral stricture. Normal flow / pathological flow.

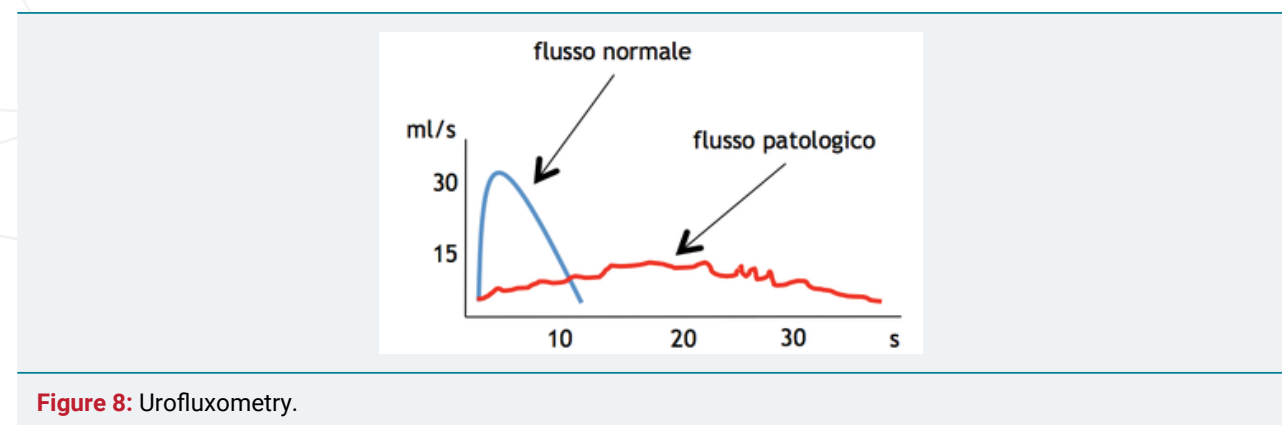

Figure 8: Urofluxometry

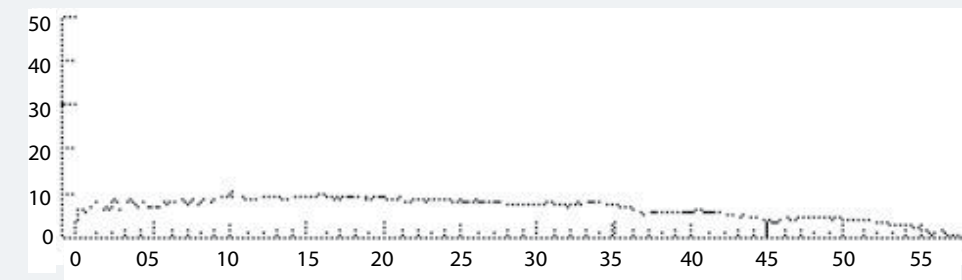

Figure 9: Show the hysto-patological changes in interstitial bladder cystitis. urehral stenosis. 


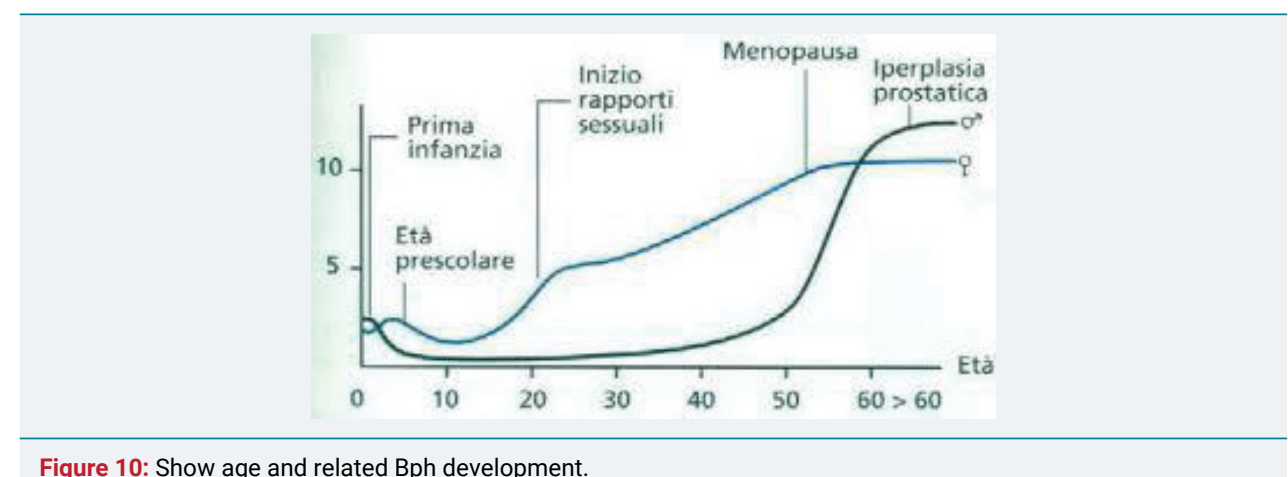

Figure 10: Show age and related Bph development.

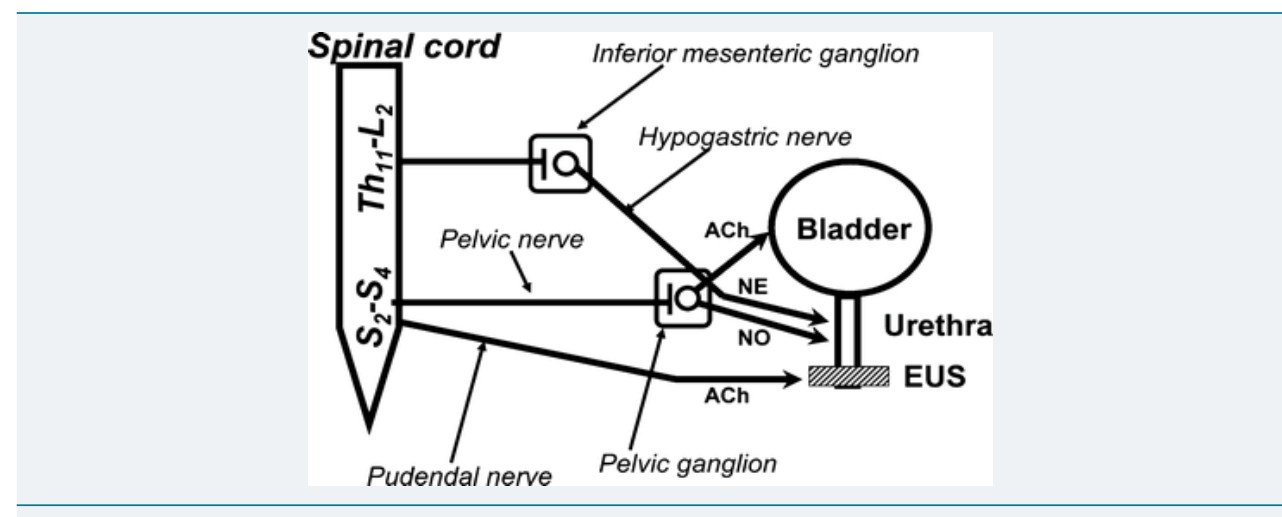

Figure 11:

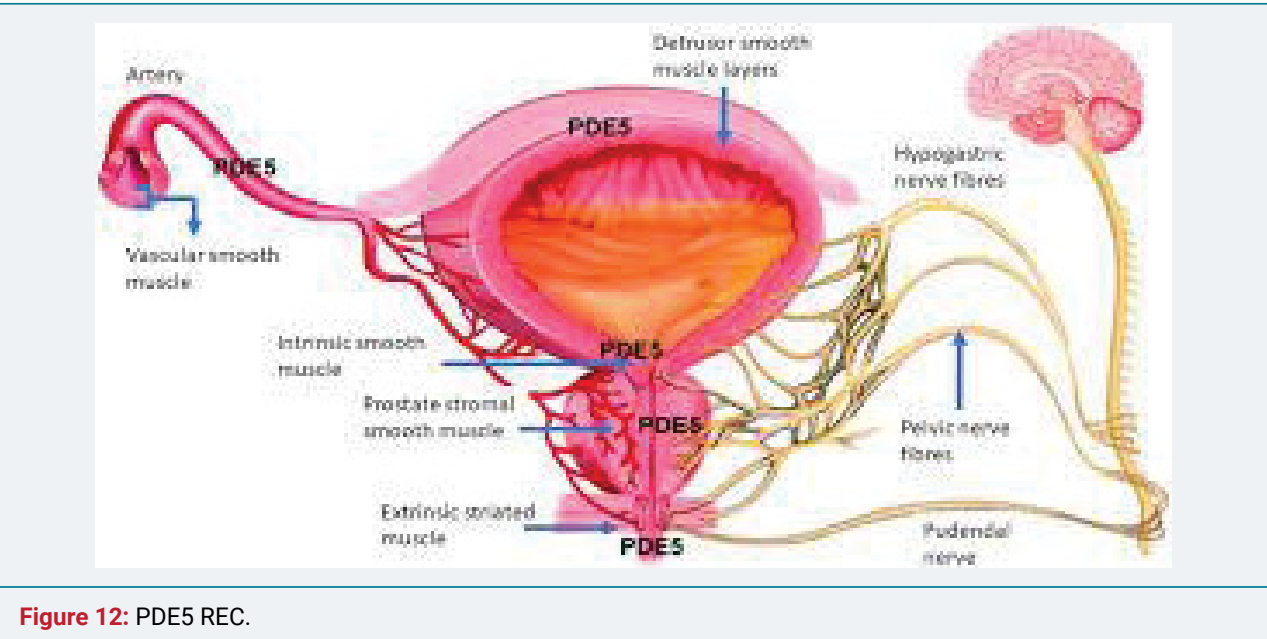

Figure 12: PDE5 REC.

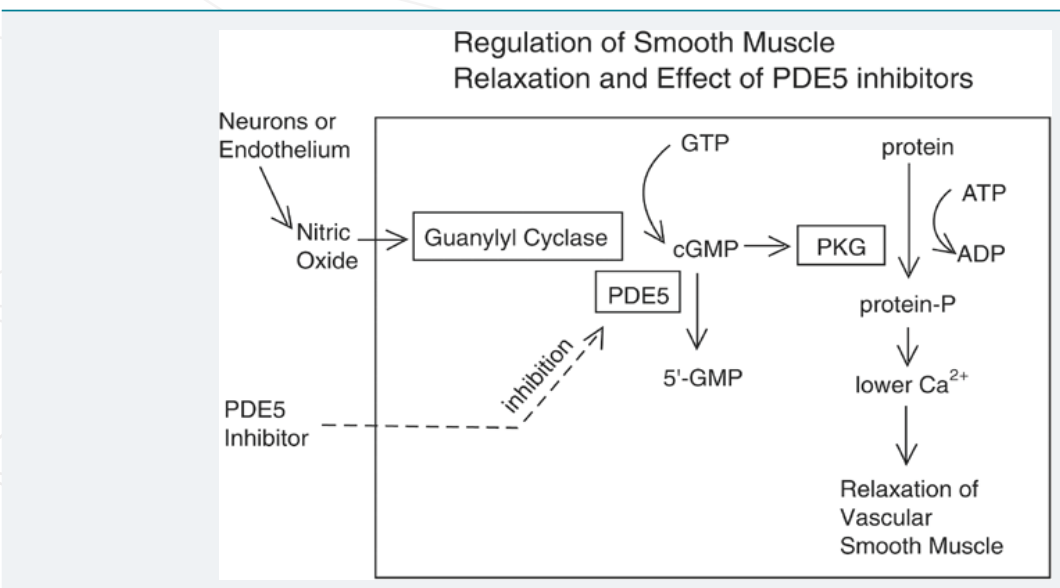

Figure 13: PDE5 physiology. 
PDE5 receptors, bladder, prostate, prostatic urethra

Other pharmacological targets: bacteria and other microorganism

Starting from the case of chronic prostatitis under a UPOINTS strategy many domains are considered in a multimodal therapy and this to confirm that a complex system must be adequately managed in a global and more objective way:

In this methodology different domains are used:

- domains U urinary: anticolinergic, alfa blockers, spasmolitics

- domains I infectious: antimicrobial with high prostate penetration :chinolons (levofloxacin, ciprofloxacin), macrolides, aminoglycoside, fosfomycin.

- domains P psyco-social: anxiolitics, antidepressive drugs, and related phytoterapic

- domains N neurologic: drugs vs neuropathic pain, or vs IBS irritable bowel syndrome

- domains 0 organ-specific: anti-flogosys, analgesic, Sereoa repens, antioxidants lycopene, selenium

- domains T: muscle tension: physical therapy, muscle-relaxant, BDZ low doses

- domains S sexual disfunctions: vardenafil, tadalafil is possible to observe how many receptor and systems are involved in a single kind of LUT condition.

In this work some biomedical article and publication are analyzed under a receptor approach in order to have a complete picture of physiology- pathology but also the complex system involved.

\section{Materials and Methods}

With an observational approach and using PUB MED database various articles are analyzed to verify The receptor - pharmacology profile of Lower urinary tract, diet influence and some behavioral habits useful to deeply understand the vicous circle involved in some LUT condition and to produce new therapeutic strategy hypothesys To improve the clinical outcomes related.

After this new research hypothesys is reported related to a functional fluxometry

To evaluate drugs efficacy in the single patients.

\section{Results}

From literature useful to this work:

According Kogan P et al., "Patients with interstitial cystitis-bladder pain syndrome IC/BPS can potentially develop symptom flares after the exposure to minor- bladder irritants such as subclinical bacterial infection. To reproduce this kind of symptom, we intra-vesically instilled a sub-noxious dose of uro-pathogenic E. coli- component lipopolysaccharide LPS in young URO-OVA/OT-I mice, a transgenic auto-immune cystitis model that spontaneously develops bladder inflammation at $\geq 10$ weeks of age. Female URO-OVA/OT-I mice 6-weeks old were treated intravesically with phosphatebuffered saline (PBS) or PBS containing a sub-noxious dose $(1 \mu \mathrm{g})$ of the LPS. Mice were evaluated for bladder inflammation, pelvic pain, and voiding dysfunction at days 1,7 , and 14 post-treatment. The Mice treated with LPS but not PBS developed early bladder- inflammation with increased macrophage- infiltration. The inflamed bladders expressed an increased levels of mRNA for proinflammatory cytokines IL-1 $\beta$ 
and IL-6 and pain mediator (subst. P precursor). LPS-treated mice exhibited pelvic pain and voiding dysfunction such as increased urinary frequency and reduced the bladder capacity. This results indicate that a single sub-noxious dosage of intravesical LPS triggers early bladder inflammation and symptom onset in URO-OVA/OT-I mice, providing a useful model for IC/BPS symptom flare study" [1].

Fiander N, "Painful bladder syndrome (PBS) and interstitial cystitis (IC) are associated with bladder pain, increased urinary frequency, urgency and reduced quality of life. The cause is still unknown, although there are several possible hypotheses. PBS/IC may significantly impact all areas of a patient's life as they try to manage their symptoms. Treatment options involve diet and fluid modifications, oral medications, intravesical therapy or as a last resort, surgery. The bladder is protected by a mucous layer known as the GAG layer and is thought to protect the underlying urothelium from coming into contact with potassium ions and other irritants contained in urine. Due to research suggesting that sufferers of PBS/IC have dysfunctional GAG layers, there is rationale for intravesical GAG replacement therapy as a treatment option in reducing PBS/IC symptoms such as pain, frequency and urgency. Early diagnosis and treatment may lead to better long-term outcomes" [2].

Wyman JFet al., showed that: "Behavioural interventions are effective treatments for overactive bladder (OAB) and urgency urinary incontinence (UUI): patient education on healthy bladder habits and lifestyle modification : establishment of normal voiding intervals, elimination of bladder irritants from the diet, management of fluid intake, weight control, management of bowel regularity and smoking cessation. Behavioural interventions include specific training- techniques aimed at re-establishing normal voiding intervals and continence. Training techniques include bladder training, which includes a progressive voiding schedule together with relaxation and distraction for urgency suppression, and multicomponent behavioural training, which, in conjunction with pelvic floor muscle (PFM) exercises, includes PFM contraction to control urgency and increase the interval between voids" [3].

Kaddumi EG et al., "The presence of pelvic visceral inputs to neurons in the rostral medulla that are responsive to electrical stimulation of the abdominal branches of the vagus nerve (VAG-abd) was investigated in a complete chronic T8 spinal transection rat model. Using extracellular electrophysiological recordings from single medullary reticular formation (MRF) neurons, 371 neurons in 15 rats responsive to pinching the ear (search stimulus) were tested for somato-visceral and viscero-visceral convergent responses to stimulation of the following nerves/territories: VAG-abd, dorsal nerve of the penis, pelvic nerve, distention of urinary bladder and colon, penile stimulation, urethral infusion, and touch/pinch of the entire body surface. In addition a chemical stimulus applied to the bladder was assessed as well. Of the total neurons examined: 205 were tested before and 166 tested beginning 20 min after application of a chemical irritant ( $2 \%$ acetic acid) to the urinary bladder (same rats used pre/post irritation). As with intact controls, many ear-responsive MRF neurons responded to the electrical stimulation of VAG-abd. MRF neuron responses failed to be evoked with direct (mechanical and electrical nerve) pelvic visceral stimuli, acute chemical irritation of the urinary bladder produced a significant increase in the number of MRF neurons responsive to stimulation of VAG-abd. The results of this study indicate a central Effect that potentially relates to some of the generalized below level pelvic visceral sensations documented in patients with complete spinal cord injury" [4].

Shorter B et al. showed, "Some Anecdotal evidence suggests that patients with painful bladder- syndrome/interstitial cystitis report symptom exacerbation after consuming particular foods, beverages and/or supplements.

Of the surveyed patients with painful bladder syndrome/interstitial cystitis about the $0.2 \%$ indicated that the consumption of certain foods or beverages caused symptom 
exacerbation. Patients who reported that specific foods worsened symptoms tended to have higher O'Leary-Sant interstitial cystitis symptom index and problem index, and/ or pelvic pain and urgency/frequency patient symptom scale scores.

35 comestible items had a mean score of lower than -1.0, including caffeinated, carbonated and alcoholic beverages, certain fruits and juices, artificial sweeteners and spicy foods.

There is a large cohort of patients with painful bladder syndrome/interstitial cystitis in whom symptoms are exacerbated by the ingestion of specific comestibles. The most frequently reported and the most bothersome comestibles were coffee, tea, soda, alcoholic- beverages, citrus fruits and juices, artificial sweeteners and hot pepper" [5].

According Ellenbroek JH et al., "To explore whether the bladder hypertrophy consistently seen in rats upon streptozotocin injection also occurs in other animal models of type 1 - 2 diabetes and how hypertrophy is linked to functional alterations of the urinary bladder.

Most animal models of diabetes exhibit less bladder hypertrophy as compared to streptozotocin-injected rats. This is not linked to type 1 vs 2 diabetes models, and type 2 models with comparable elevation of blood glucose may exhibit strong or only minor hypertrophy. Bladder dysfunction is frequently observed in experimental diabetes and mostly manifests as increased compliance but does not segregate with hypertrophy. It may at least partly reflect the need to handle large amounts of urine in models associated with major elevation of blood glucose" [6].

Tong YC et al., "This study investigated the role of sorbitol, metabolic product of glucose, in the pathogenesis of rat diabetic cystopathy. Three-month-old male Wistar rats were divided into 4 groups: 1) normal controls; 2) rats rendered diabetic by streptozotocin; 3) rats fed with glucose; and 4) rats injected with sorbitol. The M(2) muscarinic receptor (M(2)-mAChR) protein and mRNA densities of the bladder tissue were measured by Western immunoblot and Northern blot, respectively. The streptozotocin-induced diabetic rats were then treated with ONO-2235, an aldose reductase inhibitor. The bladder $\mathrm{M}(2)-\mathrm{mAChR}$ protein and mRNA were compared between the treated and untreated diabetic rats. The densities of M(2)-mAChR protein anmRNA in the bladder tissue were significantly increased in diabetic rats, and rats given either glucose or sorbitol (increases in receptor protein: $27.3+/-3.3,19.8+/$ 2.3 , and $18.0+/-2.1 \%$; increases in mRNA: $39.6+/-3.7,33.1+/-2.9$, and $20.2+/-$ $2.2 \%$, respectively). When diabetic rats were treated with $0 \mathrm{NO}-2235$, the increases in bladder M(2)-mAChR protein and mRNA were significantly alleviated. The findings suggest that sorbitol plays a role in the pathogenesis of diabetic cystopathy in rats rendered diabetic by streptozotocin. So Aldose reductase inhibitors may be useful in the treatment and prevention of diabetic cystopathy' [7].

Liu BK et al writed., "To identify the effects of the neurokinin-1 receptor (NK1R)antagonist aprepitant in treating pelvic pain, micturition symptoms, bladder inflammation in mice with an experimental auto-immune cystitis (EAC) similar to the bladder pain syndrome/interstitial cystitis (BPS/IC). The Female- C57BL/6 mice were divided into the following 3 groups: normal control, EAC, and EAC plus aprepitant. EAC was induced in mice by duplicate- immunization with bladder homogenate. In the EAC model group, EAC mice were given PBS by gavage once a day during the four week. In the EAC plus aprepitant group, aprepitant was administered instead of the PBS in the same way. After four weeks, pelvic pain threshold and urination habits of mice were analyzed, the bladder weight to body weight ratio, and histologic assessment of the expression of IL- $1 \beta$, TNF- $\alpha$, intercellular adhesion molecule 1 (ICAM-1), and NK1R in bladder tissue. EAC mice mimicked the phenol-type and the patho-physiologic 
lesions of BPS/IC well. Compared to the PBS-treated EAC mice, the mice treated with aprepitant exhibited higher pain threshold values, less number of total urine spots or small urine spots, lower bladder weight to body weight ratio, and reduced bladder inflammation with less mast cell infiltration and decreased expressions of IL-1 $\beta$, TNF- $\alpha$, and ICAM- 1 in bladder tissue. There was no- difference in NK1R expression in bladders treated with or without aprepitant. The NK1R antagonist aprepitant relieved pelvic pain, urinary symptoms, and bladder inflammation in EAC mice. This indicated that NK1R may be a novel therapeutic target in BPS/IC treatment" [8].

Butler DSC etal., "The nervous- system is engaged by the infection, indirectly through the inflammatory cascades or by bacterial attack on nerve cells. We identify a neuroepithelial activation loop that participates in the control of mucosal- inflammation and pain in acute cystitis. We show that infection activates Neurokinin-1 receptor (NK1R) and Substance P (SP) expression in nerve cells and in bladder epithelial cells in vitro and in vivo in the urinary bladder mucosa. Specific innate immune response genes regulated this mucosal response, single gene deletions resulted either in protection (Tlr $4 \%$ and Il1 $\mathrm{b}^{-\%}$ mice) or in accentuated bladder- pathology (Asc ${ }^{-/}$and $\mathrm{Nlrp}^{-\%}$ mice), compared to thecontrols. NK1R/SP expression was lower in Tlr4 $\%$ and $\mathrm{Il}_{1} \mathrm{~b}^{-/}$mice than in the C56BL/6WT controls but in Asc ${ }^{--}$and Nlrp3 ${ }^{-/}$mice, NK1R over-activation accompanied the exaggerated disease phenotype, due, in part to transcriptional derepression of Tacr1. Pharmacologic NK1R -inhibitors attenuated acute- cystitis in susceptible mice, supporting a role in disease pathogenesis. Clinical- relevance was suggested by elevated urine SP- levels in patients with acute cystitis, compared to patients with asymptomatic bacteriuria identifying NK1R/SP as potential therapeutic targets. We propose that the NK1R and SP influence the severity of acute cystitis through a neuro-epithelial activation loop that controls pain and mucosal inflammation' [9].

Tourret J et al., "Urinary tract infections occur more frequently in diabetic patients than in the general- population, with a relative risk ranging from $1.5-4$, depending on excre type of infection. Urine glucose excression (which could facilitate the bacterial urinary proliferation), immunodeficiency, a modified urothelium (resulting in a higher bacterial adhesion), and chronic -neurologic bladder dysfunction have been advocated. Diabetes mellitus has recently been withdrawn from the list of the criteria which define an urinary- tract infection as complicated" [10].

Ross MA., "Autonomic- neuropathy is a frequent feature of diabetic neuropathy and the source of many significant problems including postural hypotension, gastroparesis, diarrhea, constipation, neurogenic bladder, and male impotence. The cause of the diabetic- neuropathy is not determined, even abundant evidence suggests that both metabolic and ischemic nerve injury are likely factors. Although no effective treatment exists to cure diabetic neuropathy, improvement is possible with glycemic control and symptomatic therapy" [11].

"Chronic elevation in cytosolic glucose leads to metabolic abnormalities such as osmotic and oxidative stress, thought to be factors that contribute to tissue injury and dysfunction associated with long-term DM.

The urinary bladder is one of the many organs affected by DM. Urine production is increased as hyperglycemia results in elevated glucose filtered load, which when exceeding the reabsorption capacity (renal threshold) of the kidney, leads to osmotic diuresis. Initially the bladder adapts to polyuria by compensatory, increased activity but subsequently decompensates due to the direct effects of chronic systemic hyperglycemia on bladder tissues. DM patients commonly fail to recognize urological symptoms and thus treatment strategies are more likely to be applied at a late stage.

Diabetic cystopathy/diabetic bladder dysfunction (DBD) is a very common DMassociated lower urinary tract pathology, occurring in almost $80 \%$ of patients. It is 
a complex-multifactorial disease that affects both storage and emptying/voiding functions and is characterized by a broad spectrum of symptoms: urinary urgency, frequency, nocturia, and incontinence [12].

Though the urothelium maintains a tight barrier to ion and solute flux, a number of local factors: tissue $\mathrm{pH}$, mechanical or chemical trauma, or bacterial infection can modulate the barrier function of the urothelium. When the barrier is compromised, water, urea, and toxic substances can pass into the underlying tissue (neural/muscle layers) resulting in urgency, frequency, and pain during the bladder filling and voiding. Disruption of urothelial barrier integrity has also been linked to the expression of substances: antiproliferative factor (APF), which also slows urothelial cell growth.18-20 APF, a frizzled 8 protein detected in the urine of patients with bladder pain syndrome" [13].

According Thor KB et al., "the role of 5-HT(1A) receptors in control of lower urinary tract function in cats was examined using 8-hydroxy-2-(di-n-propylamino)tetralin (8-OH-DPAT) and 5-methoxy-N,N-dimethyltryptamine (5-MeODMT) as agonists and WAY100635 and LY206130 as antagonists. Bladder function was assessed using a cystometric- infusion of saline solutions or $0.5 \%$ acetic acid to produce bladder- irritation. External urethral sphincter (EUS) function was assessed using an electromyographic a (EMG) recordings of activity recorded during cystometry or by recording electrically evoked a pudendal reflexes. 5-HT(1A) receptor agonists caused dose-dependent decreases in bladder activity and increases in EUS EMG activity under conditions of an acetic acid infusion. The 5-HT(1A) rec. antagonists reversed both bladder-inhibitory and sphincter facilitatory- effects. 5-HT(1A) receptor activation can have opposite effects on the nociceptive afferent- processing depending upon the efferent response being measured. During the saline solutions infusion of the bladder, 8-OH-DPAT produced moderate inhibition of bladder activity and had no significant effect on sphincter electromyographic EMG activity. 8-OH-DPAT either had no effect or inhibited low-threshold electrically evoked pudendal reflexes. These indicate that 5 -HT(1A) receptor stimulation is inhibitory to the bladder function in cats, especially under conditions where the bladder is hyperactive due to irritation. These bladderinhibitory effects are the exact opposite of the bladder-excitatory effects of 8-OH-DPAT reported in rats. 5-HT(1A) receptor stimulation increases EUS motoneuron activity when driven by nociceptive bladder afferent inputs but not when driven by a non-nociceptive afferent inputs. In summary, 5-HT(1A) receptor agonists facilitate a nociceptor-driven spinal reflex (sphincter activity) but inhibit a nociceptor-driven supra-spinal reflex (micturition). This pattern of activity would facilitate the urine storage and may be important under 'fight-or-flight' conditions when serotonergic activity is high" [14].

"BPH is currently consider a common- progressive enlargement of the prostate gl caused by benign overgrowth of chiely glandular tissue that occurs especially in some men $>50$ years old and that tends to obstruct urination by constricting the urethra with heavy symptomatology and related consequences in quality of life. The current pharmacological therapy (based on 5-ARI, alfa blokers, antimicrobials,fitoth erapics,anti Flogosys Anti-edemigen and other) can and must be improved to delay or avoid surgery. To introduce new- pharmacological strategy is crucial to observe the immune implications in BPH. Reports in The biomedical litrerature database indicate that in BPH progression some of the following factors are heavily involved: Bacterial/ viral prostate infections, chronic phlogosis, hormonal factors,. Autoimmune reactions, Urinary relux, Diet, Seating bathroom habits/behavior, sedentariety,. Pelvic congestion,. Proctities, Prostate ducts obstructions, microcalcifications.

All this facts contribute to an increased production of phogosis mediators growth factors (stromal and epithelial) in a vicious cycle and as a result, increase glands and complication of the pathogenic- conditions. The presence of immune- system in prostate prostate tissue is very well alive and manifest itself in different forms, from 
an iniltration of lymphocytes $\mathrm{T}$ and macrophages caused by phlogosis to cytokines that cause hypertrophy. These reactions along with the cellular damage produce ROS reactive oxygen species, increase in production of growth factors (like VGEF, IL 8 FGF 7, TGFB, FGF 2 and others), inhibition of apoptosis and tissue remodelling with hyperproduction of extracellular matrix and stromal parts. Other factor that can be responsible in the relapse in chronic prostatitis in BPH are micro-calciications, obstruction of the ducts. But there are still other conditions that can be involved, such as metabolic syndrome, diabetes and the related biochemical and hormonal factors that can modify the methabolisms that can worsen the pathogenesis. Other factors still play in. Ex. the command-/-control of the nervous systems over the bladder, uretral restrictions and other Urological disease can cause important complications. The creation of a biofilm over the prostate capsule gland makes the penetration of several antimicrobial agents look like resistant which reduces their prostatic inlux. Be patient it's not over yet, we can still mention the complications caused by such as IPB, urinary relux (chemical cystitis and prostatitis), prostate cancer and other factors. Often Intestinal functional abnormalities and pathologies, anorectal, sexually- transmitted infectious as complication due to bacterial sanctuaries and intrapostatic calcifications.

A bacterial infection can be primary or secondary (bacteria that causes infections or super-infectious in a tissue affected by phlogosis or to other conditions), for example in hypertonic pelvic sphincter- muscle. (Bacteria can come across also the lymphatic way (from rectum), by emetic way, rising uretral infectious). Adrenergic- ipertonus of prostate capsule contribute to BPH physio-pathology. Involved often intestinal functional abnormalities and pathology' [15].

"It is known that the prostate is frequently involved in various pathologies in adults and elderly patients. Benignant o/malignant: anatomic of functionally disease. Frequently other condition like bladder dysfunctions can be associated or added to this pathologies or causated by it. Some of these pathologic conditions give low level in patient quality of life and reducing in life expectance (malignant). Malignant pathologies even start as local disease, but can diffused as metastatic interesting other apparatus of the patient (patient frequently show resistance to first line therapy in example hormonal blocks, or different chemiotherapic).

But in this pathology we can see a not complete resolution with current medical therapy in all patients and this can be related with the difficulties of some drugs molecules to penetrate adequately in the tissue. In ex. I many benignant cases as IPERTROPHIA also fitotherapic produces or dietetic integrator are frequently added by the urologist to the classic drugs politherapy.

This fact shows that the classic drug therapy can be improved. In Relapses in bacterial chronic prostatitis are commonly in great number of patient, even under the best pharmacological therapies available today. It means that something goes wrong in this pharmacological therapy.

About bacterial chronic prostatitis we can see that some antimicrobials are used for long time cycle in order to have efficiently concentration of the drugs in a sanctuaries tissue as prostatic but this not gives a healing to the entire patient treated and we can have toxicity not irrelevant. We can have different problems: Antimicrobial activity to cover the bacteria spectrum and the concentration active in the tissue for the right time necessary (Dynamic and kinetics).

So the answer today is: Have the right drugs today, need we knew delivery system to produce rely ERLICH MUGIC BULLET expecifically for prostatic tissue? We think that this clinical and therapeutic need in underestimate by pharmaceutical industries (our opinion). Why is today accepted to give great amount of antimicrobial to cure this kind of disease with high number of relapses (augmented toxicity for the patient and diffusion 
of resistances). Analyzing the literature we can see that adding the clinical pharmacist competence in many medical teams we can have global increase in clinical outcomes.

We can think that the same we can have in some pathology with high relapses as chronic prostatitis but also in malignant cases (chemiotherapic and other class of molecule available today) we can see that different chemio-therapic drugs are used but patient with resistance can die by metastatic disease (Different drugs, with different way of action). If we know that there is a problem of ph. Kinetics and dynamics of drugs we must clearly say that we need a really new approach to make possible the drug's efficacy act in site of actions as request for an efficiently therapy.

It is clear that the drugs vehicle is important as the same drugs and that modifying the molecule we can improve the activity in relevant way also due in example a different tissue diffusion (In example in firt chinolons vs fluorochinolons).

Lipophilic- hydrophilic balances great influence the pk. Kinetics, VD or $\mathrm{T}_{1 / 2}$ but also the profile of linked chemical groups can give new delivery of classic drugs (Other can be acid or basic molecular properties or velocity in metabolism). In ex. see the activity of some MABS linked with radio isotopes in order to send the radioactivity in specific target reducing the complex toxicity. The specific Expertize of clinical pharmacy added to the medical team and to the pharmaceutical industries can help in finding new efficacy solution in drug delivery.

Medicinal chemistry knowledge added to advanced pharmacokinetics and dynamics, metabolism and Toxicology of clinical pharmacist can drive the pharmaceutical industries in the right direction.

The same a deep knowledge in resistance mechanism (drugs extrusion from intracellular and other) can be a great instrument in drugs design. In ex. it is showed that some intracellular chemiotherapic are extruded by the cell like a normal poison. The same genomic mechanism of resistances and modified enzymatic level, target modify, can make the difference in clinical outcomes. Observing some relevant biomedical literature we can see that if in acute situation we have seen a positive impact in improving clinical outcomes as in ICT medical team due by active participation of the clinical pharmacist we can have same improvement also in chronic condition [16].

"According Bio-medical Literature published responsible of relapses of chronic prostatitic and relapses can be: biofilm, reduced urinary prostatic fluss in example: by IPB, urinary reflux (chemical cystitis and prostatitis), prostatic cancer et other factors" [17].

XuZetal.,"Evidence confirmed thatBPH/LUTS is strictly related to erectile dysfunction (ED) and significantly affects the quality of life of elderly males. Phosphodiesterase 5 inhibitors (PDE5i) can improve both ED and BPH/LUTS of the patients and PDE5 is expected to be a new therapeutic target for BPH/LUTS with ED" [18].

Magri V et al., "The complex network of etiological factors, signals and tissue responses involved in chronic prostatitis/chronic pelvic pain syndrome (CP/CPPS) cannot be successfully targeted by a single therapeutic agent. Multimodal- approaches to the therapy of CP/CPPS have been and are currently being tested, as in the frame of complex diagnostic-therapeutic phenotypic approaches such as the urinary, psychosocial, organ-specific, infection, neurological and muscle tenderness (UPOINTS) system.. Patients received $\alpha$-blockers, Serenoa repens extracts combined or not with supplements (lycopene and selenium) and, in the presence of documented or highly suspected infection, antibacterial agents. Combination treatment induced marked and significant improvements of National Institutes of Health Chronic Prostatitis Symptom Index (NIH-CPSI) prostatitis symptom scores, International Index of Erectile Function (IIEF) sexual dysfunction scores, urinary peak flow rates and bladder voiding efficiency. A clinically appreciable reduction of $\geq 6$ points of the total NIH-CPSI score 
was achieved in $77.5 \%$ of patients subjected to combination therapy for a period of 6 months. When the patients were divided in 3 cohorts, depending on the diagnosis of CP/CPPS [inflammatory (IIIa) vs. non-inflammatory (IIIb) subtypes], significant improvements of all signs and symptoms of the syndrome were observed in both cohorts at the end of therapy.A simple protocol based on $\alpha$-blockers, $S$. repens extracts and supplements and antibacterial agents, targeting the urinary, organ specific and infection domains of UPOINTS, may induce a clinically appreciable improvement of the signs and symptoms of CP/CPPS in a considerable percentage of patients. In patients not- responding sufficiently to such therapy, 2-line agents (antidepressants, anxiolytics, muscle relaxants, 5-phosphodiesterase inhibitors and others) may be administered in order to achieve a satisfactory therapeutic response" [19].

Ozaki T et al., "Ca $3.2 \mathrm{~T}$-type $\mathrm{Ca}^{2+}$ channel activity is suppressed by zinc that binds to the extra-cellular histidine-191 of $\mathrm{Ca}_{\mathrm{v}} 3.2$, and enhanced by $\mathrm{H}_{2} \mathrm{~S}$ that interacts with zinc. $\mathrm{Ca}_{\mathrm{v}} 3.2$ in nociceptors is upregulated in an activity-dependent manner. The enhanced $\mathrm{Ca}_{\mathrm{v}} 3.2$ activity by $\mathrm{H}_{2} \mathrm{~S}$ formed by the upregulated cystathionine- $\gamma$-lyase (CSE) is involved in the cyclophosphamide -induced cystitis-related bladder pain in the mice. We thus asked if the zinc deficiency affects the cystitis-related bladder pain in mice by altering $\mathrm{Ca}_{\mathrm{v}} 3.2$ function and/or expression. Dietary zinc deficiency for 2 weeks greatly decreased zinc concentrations in the plasma but not bladder tissue, and enhanced the bladder pain/referred hyperalgesia (BP/RH) following CPA at $200 \mathrm{mg} / \mathrm{kg}$, a subeffective dose, but not $400 \mathrm{mg} / \mathrm{kg}$, a maximal dose, an effect abolished by pharmacologicalblockade or gene silencing of $\mathrm{Ca}_{\mathrm{v}} 3.2$. Together, zinc deficiency promotes bladder pain accompanying CPA-induced cystitis by enhancing function and expression of $\mathrm{Ca}_{\mathrm{v}} 3.2$ in nociceptors, suggesting a novel therapeutic avenue for treatment of bladder pain, such as zinc supplementation" [20].

Lovick TA., "Afferent input from A $\delta$ and C-fibres innervating the urinary bladder are processed differently by the brain, and have different roles in signaling bladder sensation. A $\delta$ fibres that signal bladder filling activate a spino-bulbo-spinal loop, which relays in the midbrain periaqueductal grey (PAG) and pontine micturition centre (PMC). The excitability of this circuitry is regulated by tonic GABAergic inhibitory processes. In humans and socialised animals micturition is normally under volitional control and influenced by a host of psychosocial factors. Higher nervous decisionmaking in a social context to 'go now' or 'do not go' probably resides in frontal corticalareas, which act as a central control switch for micturition. Exposure to psychosocial stress can have profoundly disruptive influence on the process and lead to maladaptive changes in the bladder. During sleeping the voiding reflex threshold appears to be reset to a higher level to promote urinary continence. Under physiological conditions C-fibre bladder afferents are normally silent but are activated in inflammatory bladder states and by intense distending pressure. Following prolonged stimulation visceral nociceptors sensitise, leading to a lowered threshold and heightened sensitivity. Sensitization may occur within the central- pain processing circuitry, which outlasts the original nociceptive insult. Visceral nociception may also be influenced by genetic and environmental influences. A period of chronic stress can produce increased sensitivity to visceral pain that lasts for months. Adverse early life events can produce even longer lasting epigenetic changes, which increase the individual's susceptibility to developing visceral pain states in adulthood" [21].

Tonon Let al., "Causes of high mortality of prostate cancer in men of African ancestry living in the French-West Indies are still debated, (suspicions of environmental factors and the genetic susceptibility). The results show that African- Caribbean tumours are characterised by a more frequent deletion at 1q41-43 encompassing the DNA repair gene PARP1, and an higher proportion of intrachromosomal rearrangements including duplications associated with CDK12 truncating- mutations. Transcriptome- analyses show an overexpression of genes related to androgen receptor- activity in African 
Caribbean tumours, and of PVT1, a long non-coding RNA located at 8q24, this confirms the high involvement of this region in prostate tumours from men of African ancestry. Mortality by prostate cancer is higher in African Caribbean men than in French Caucasian men. Specificities of the former could be explained by genomic -events linked with key genes such as DNA- damage pathway genes PARP1, CDK12, and the oncogenic long non coding RNA gene PVT1 at the 8q24 prostate cancer susceptibility locus" [22].

Firouz Daneshgari et al., "In natural history of DBD, we have observed that morphological- functional manifestations of DBD in STZ-induced DM are timedependent. Bladder hypertrophy and remodeling, increased contractility and associated neurogenic -changes occur soon after the onset of DM. While the drop of peak voiding pressure in the cystometric- measure, develop only at a later stage of DM. The timedependent alterations of DBD served as the basis for our 'temporal hypothesis' of DBD with mixed clinical manifestations, in which we propose that DM causes the bladder to undergo 2 phases of alterations via two main mechanisms: In the earlyphase, hyper-glycemia-induced osmotic polyuria is the main mechanistic factor that causes compensatory bladder hypertrophy and associated myogenic and neurogenic alterations. Later phase, accumulation of oxidative- stress products during prolonged hyperglycemia causes decompensation of the bladder tissues and function.

Unlike most other organs affected by D.Mellitus, the bladder faces not only hyperglycemia, but also an exceptionally high volume of urine output. In the experimental models, sucrose-induced diuresis causes rapid and substantial bladderhypertrophy and increased bladder contractility, capacity and compliance that are similar to those changes observed in the diabetic rats. Those similarities suggest that bladder hypertrophy in diabetic- animals may result from a physically adaptation to increased urine production. Bladder hypertrophy may also initiate the process of the increased oxidative stress.

The excess ROS -cause, in turn, DNA strand breaks, activation of poly (ADP-ribose) polymerase PARP and inhibition of glyceraldehyde-3 phosphate dehydrogenase (GAPDH), culminating in the activation of the four damaging pathways.

Numerous studies in the role of oxidative stress on the pathogenesis of diabetic complications in the eye, nervous -system, kidney and cardiovascular system, studies on the direct effect of oxidative stress in the urologic -complications has not yet been investigated in detail. Few studies on its role in erectile -dysfunction and on cystopathy have indicated the importance of oxidative stress in the pathogenesis of urologic diabetic -complications. Using rabbit model of alloxan-induced diabetes, we showed that the decrease in the contractility of the detrusor smooth muscle (DSM) is associated with increased lipid peroxidation products and over-our preliminaexpression of aldose reductase. Mitochondria are the major source of superoxide, peroxynitrite and hydroxyl radicals in all types of cells. Our data show that treatment of high glucose increases the mitochondrial membrane potential and ROS in cultured human bladder smooth muscle cells (hBSMC), in agreement with the published reports showing that the mitochondrial dysfunction is a key mechanistic step in diabetes complications. Mitochondrial dysfunction reduces the production of ATP, affecting the ability of cross -bridges to cycle during the force generation.

Endogenous antioxidants are able to destroy the ROS and create a balance between antioxidant and free radicals in a normal situation; however, in diabetes the antioxidant defense system is deficient due to the high level of oxidative stress. Intake of the antioxidants, such as vitamin E and $\alpha$-lipoic acid, which functions as a cofactor in multi-enzyme complexes, have been successfully used to reverse oxidative stress produced by hyperglycemia in human diabetics and in STZ-induced diabetic animal models. An Oral treatment of $\alpha$-lipoic acid (600 mg / day for 5 weeks) improved neuropathic deficits in diabetic patients with distal symmetric polyneuropathy in a recent clinical trial. 
Pathophysiology of DBD is multifactorial: it involves alterations in detrusor, nerve, urothelium and urethra.

The pathophysiology of DBD is multifactorial, including disturbances of the bladder detrusor, urethra, autonomic nerves, and perhaps the urothelium. Researcher have observed that, upon induction of DM in the rodents by destruction of the pancreatic $\beta$-cells with STZ, the bladder and urethra undergo morphometric and functional changes in both myogenic - neurogenic components. Other study has demonstrated the potentially obstructive effects of urethral sphincteric mechanisms in DBD.

In vivo and in vitro experimental studies on DSM from animal models of DM do give evidence for myogenic changes. Studies on the effects of diabetes on detrusor contractility show decreased and increased force production in rat DSM strips. Contractility of the DSM was decreased in response to stimulation by $\mathrm{KCl}$ and carbachol, and the decrease was associated with not only the duration of the hyperglycemic state but also the level of hyperglycemia. Changes in muscarinic -receptor population have also been linked to altered contractility. Unlike the changes in the DSM from an obstructed bladder, we found, using STZ-induced rat diabetic model and an alloxaninduced rabbit model, that there are no change in the myosin isoform composition in the DSM from diabetic animals.

A > regulatory mechanism for smooth muscle contraction is the myosin-mediated regulation via phosphorylation-dephosphorylation of the regulatory myosin light chain $\left(\mathrm{MLC}_{20}\right)$ by $\mathrm{Ca}^{2+}$-dependent myosin light chain kinase (MLCK) and the myosin light chain phosphatase (MLCP). The MLCP is inactivated by phosphorylation (catalyzed mainly by Rho-kinase)rho-, and also by binding to phosphorylated CPI-17. By lowering the activity of the MLCP, these proteins retain the myosin in the phosphorylated state and maintain the muscle tone in the absence of an elevation of cytosolic $\mathrm{Ca}^{2+}$. Studies performed on DSM from diabetic animals showed overexpression and over-activity of Rho-kinase and CPI-17 proteins involved in the $\mathrm{Ca}^{2+}$-sensitization in smooth muscle. We also found high level of basal $\mathrm{MLC}_{20}$ phosphorylation in the diabetic- detrusor.

Other important, function of epithelial cells is their ability to sense the changes in their extracellular environment and then communicate these changes to the underlying nervous, connective and muscular tissues. This communication is likely to be very important for tube- and sac-shaped organs such as blood vessels, the gut, bladder, whose normal function can be modulated by stimuli initiated within the epithelium. Though alterations in smooth muscle and nerve innervation have been shown in diabetic patients, there are little information regarding the involvement of the urothelium in the pathophysiology of DBD.

Studies using the STZ-induced diabetic rat model, report an increase in urothelium proliferation without an increase in the thickness of the urothelial lining itself. This increase in the proliferation may divert the physiology of the urothelium cells from their normal inter-communication/2-way communication with the underlying bladder tissue, by modifying both urothelial cell -receptor expression and the release of signaling molecules such as neurotransmitters. This in turn could impact/modify the activity in underlying smooth muscle and nerve endings and contribute to the bladder function modification observed in DM. It was reported that urothelial cell prostaglandin- release is impaired, in STZ-DM rats. This might affect the barrier function of the urothelium. Prostaglandins are known to play an important role in the maintenance of musical integrity in the gut. In addition it has been proposed that the common occurrence of urinary tract infections seen in DM and attributed in part to the bladder stasis seen in the pathology, may be as a result of altered expression of adherence receptors for bacteria by urothelial cells.

Abnormalities in bladder- urothelium could impact the LUT function by altering 
release of mediators as excitability of sensory fibers in the bladder. Many urothelial functions may be altered in diabetes, defects in urothelial cells may underlie, in part, changes such as detrusor instability and/or changes in bladder capacity.

The urothelium is an active participant in the normal function of bladder and exists as an integral part of a sensory web, in which it communicates the degree of bladder filling to the underlying nervous and muscular tissues and affects their functions. This communication is made possible by the input / output pathways of the urothelium, which allow it to respond to its chemical and physical environment and to engage in multi-directional communication with neighboring cells in subadjacent tissues. Defects in urothelial expression of the receptors or aberrant release of mediators may contribute to diabetes-associated bladder complications.

The neuronal control bladder function involves a very complex interaction between the autonomic and somatic afferent and efferent pathways. One group reported an association of DBD with autonomic neuropathy detected by sympathetic skin response in diabetic patients. Steers et al showed significant abnormalities in the afferent pathways innervating the bladder in STZ induced diabetic rats. Adult rats that was treated with capsaicin, a C-fiber afferent neurotoxin, exhibit a number of similarities to diabetic rats. Since the capsaicin is known to affect predominately small myelinated and unmyelinated afferents, it is tempting to speculate that DM affects a similar afferent neuron population. It has been suggested that DBD is initiated by a neuropathy in the efferent limb of the micturition reflex.

Neurotrophic factors derived from target -tissues can support the growth and survival of peripheral neurons. Rats with STZ-induced DM, 12 weeks after induction, showed significantly decreased levels of nerve growth factor NGF, a the neurotrophin, in the bladder and in L6 to the S1 dorsal root ganglia, which contain bladder afferent neurons. Reports that diabetic rodents show a loss of neurotrophic support to peripheral nerves have prompted studies to investigate efficacy of neurotrophic factor supplementation on nerve disorders of diabetic rats. The use of an exogenous neurotrophic factors as a therapy is limited by the need for non-oral delivery, the fiber selectivity of individual neurotrophins, limited delivery to the nervous system and concerns about harmful systemic-actions of growth factors" [23].

Montalbetti $\mathrm{N}$ et al., showed that "Interstitial cystitis/bladder pain syndrome (IC/ BPS) is a chronic voiding disorder that presents with pain in the urinary bladder and surrounding pelvic region. Evidence suggests that: an increase in the permeability of the urothelium, the epithelial barrier that lines the interior of the bladder, contributes to the symptoms of IC/BPS. To examine the consequence of increased urothelial permeability on pelvic pain and afferent excitability, we overexpressed in the urothelium claudin 2 (Cldn2), a tight junction (TJ)-associated protein whose message is significantly upregulated in biopsies of IC/BPS patients. In presence of bladder-derived pain, rats overexpressing Cldn2 showed a hypersensitivity to von Frey filaments applied to the pelvic region. Overexpression of Cldn2 increased the expression of c-Fos and promoted the activation of ERK1/2 in spinal cord segments receiving bladder input, which we conceive is the result of noxious stimulation of afferent pathways. To determine whether the mechanical allodynia observed in rats with reduced urothelial barrier function results from altered afferent activity, we examined the firing of acutely isolated bladder sensory neurons. In patch-clamp recordings, about $30 \%$ of the bladder sensory neurons from rats transduced with Cldn2, but not controls transduced with GFP, displayed spontaneous activity. Bladder sensory neurons with tetrodotoxin-sensitive (TTX-S) action potentials from rats transduced with Cldn2 showed hyperexcitability in response to suprathreshold electrical stimulation. These findings suggest that as a result of a leaky urothelium, the diffusion of urinary solutes through the urothelial barrier sensitizes bladders afferents, promoting voiding at low filling volumes and pain" [24]. 
Helen L. Nicholson et al. writed, the aim of this review- study is to examine all urethral strictures and stenoses subsequent to treatment for prostate cancer, including radical prostatectomy (RP), radiotherapy, high intensity focused ultrasound (HIFU) and cryotherapy. The overall majority respond to the endoscopic treatment, including dilatation, direct visual internal urethrotomy (DVIU) or bladder neck incision (BNI). There are adjunct treatments to endoscopic management, including injections of corticosteroids and mitomycin- C (MMC) and urethral stents medical devices, which remain controversial and are not currently mainstay of treatment. Recalcitrant strictures are most commonly managed with urethroplasty, while recalcitrant stenosis is relatively rare yet almost always associated with bother some urinary incontinence, requiring bladder neck reconstruction and subsequent artificial urinary sphincter implantation, or urinary diversion for the devastated outlet" [25].

Zhigang Zhao et al., "Chronic prostatitis/chronic pelvic pain syndrome (CP/CPPS) present significant economic costs and severe impact on the quality of life of diagnosed patients. Prevalence: between $2.2 \%$ and $13.8 \%$. Symptoms of this condition include chronic pain, voiding symptoms, pelvic, sexual and psychosocial disturbances, among others. Until To date, no single specific therapy is effective in all the patients. The major reason for this is that the patients with CP/CPPS are not a homogenous group with a single disease process who respond in the same way to specific medications, but rather a heterogeneous group of unique individuals with widely different etiological mechanisms, disease characteristics, symptom complexes, and progression trajectories. It is rational to evaluate $\mathrm{CP} / \mathrm{CPPS}$ patients as individuals with differing clinical phenotypes. No- validated predictors /biomarkers are currently available that help classify those patients in a way that could guide the therapy. In 2009, Shoskes et al., developed a 6-point clinical phenotyping system called UPOINT to classify the patients with CPPS and interstitial cystitis and subsequently direct appropriate therapy. The clinical- domains are urinary symptoms, psychosocial dysfunction, organ specific findings, infection, neurological/systemic, and tenderness of muscles. Each domain has been clinically- defined, linked to the specific mechanisms of symptom production or propagation, and associated with the specific therapy. This kind of phenotype is qualitative, with each domain scored as yes or no. The major finding of the first UPOINT retrospective study was the strong correlation between the number of UPOINT positive domains and the NIH-CPSI total- score in each patient, which was further verified by other studies. Recently, the UPOINT-guided multimodal therapy has been shown to significantly improve symptoms.

It is widely acknowledged that CP-CPPS is associated with significant sexual dysfunction. Erectile dysfunction (ED) is the most investigated sexual dysfunction in patients with CP/CPPS. The reported ED prevalence findings for CP/CPPS sufferers ranged from $15.0 \%$ - 48.3\%. However, the original UPOINT phenotype system did not include a domain for sexual dysfunction or ED. A Swedish study recently showed that the number of positive UPOINT domains was not correlated with ED severity in CP/ CPPS patients. The impact of adding a sexual- dysfunction or ED domain to the UPOINT system on patients symptoms is also conflicting.

For international validation and adoption of this novel UPOINT algorithm a prospective study was conducted in a Chinese- cohort of males with CP/CPPS. We sought to determine the clinical- phenotype of those Chinese CP/CPPS patients using the UPOINT classification system and assessed the frequency of individual domains and their effect on symptom severity and $m$ the erectile function. We aimed to further investigate the impact of adding an ED domain to create a modified UPOINT phenotyping system on symptom severity of CP/CPPS patients

The utility of the UPOINT classification scheme in discriminating clinical phenotypes has been validated in the Chinese cohort of CP/CPPS, with the number of + domains 
shown to be strongly correlate with the duration and severity of prostatitis symptoms and with the ED symptoms. Adding an ED domain restored a significant association between the number of a modified UPOINT domains and the NIH-CPSI scores. Our prospective analyses demonstrate that ED is an important component of the clinical phenotype of CP/CPPS, the addition of an ED domain to the UPOINT- system adds a value to the clinical assessment of the CP/CPPS symptom severity. This results here support the utility of using ED as an independent UPOINT domain" [26].

Kim Moon et al., Acute ethanol intoxication is a predisposing factor for urinary retention induced by BPH. The mechanisms of ethanol-induced urinary retention are an alcohol-induced diuresis and the $\alpha$-sympathomimetic effect of ethanol itself, along with depression of the CNS. Ethanol-induced congestion of the prostate and the prostatic urethra could also obstruct outflow. After ingestion, ethanol is metabolized sequentially to acetaldehyde, acetate, $\mathrm{CO}_{2}$ and water. The negligible blood concentration of acetaldehyde found after the ingestion of alcohol in most Caucasian subjects under normal conditions suggests that acetaldehyde is of interest only when acetaldehyde dehydrogenase (ALDH2) is inhibited. Aabout half of Oriental subjects have a deficiency of mitochondrial ALDH2, resulting in increasing acetaldehyde levels after ethanol intoxication. Ethanol seems to inhibit smooth muscle contraction but the effects of ethanol and acetaldehyde on the responses of the bladder have not been assessed.

Acute ethanol intoxication cause urinary retention in patients with BPH. In animals ethanol significantly impaired detrusor contractility in vivo- in vitro. The primary enzymatic steps in ethanol metabolism to acetaldehyde, acetate, $\mathrm{CO}_{2}$ and water have been known for many decades.

Alcohol-related flushing, which occurs in about half of Oriental subjects after ingestion of alcohol, is caused by deficient activity of mitochondrial ALDH2, resulting in increased acetaldehyde levels. Acetaldehyde is the principal metabolic by-product of ethanol metabolism causing cytotoxicity, and may be limited by administering vitamin C. In an animal study, chronic acetaldehyde exposure impaired the inotropic response to membrane depolarization in endothelium-denuded preparations of rat aorta, resulting in depressed responsiveness; acute acetaldehyde exposure significantly reduced both noradrenaline- and $\mathrm{KCl}$-induced contractile responses, but low concentrations of acetaldehyde enhanced the spontaneous phasic contractile activity. In Normally circumstances, the acetaldehyde produced as a result of ethanol metabolism is rapidly oxidised to acetate by aldehyde dehydrogenase, and other microsomal enzymes. There are several conditions in which the plasma concentration of acetaldehyde is significantly increased after ethanol ingestion, e.g. alcoholism or subjects undergoing therapy with oral hypoglycaemic agents. Therefore, after alcohol ingestion, acetaldehyde may affect bladder detrusor contractility, although the effects of acetaldehyde on bladder detrusor muscle remain uncertain.

The present study investigated the effects of acetaldehyde on isolated rat bladder muscle, thus eliminating the effects of innervation. Acetaldehyde increased bethanechol-induced bladder contraction when applied acutely, although chronic exposure to ethanol and acetaldehyde alone decreased the contractility of the bladder. In the guinea-pig, atropine did not prevent acetaldehyde-induced bronchoconstriction, and acetaldehyde has a bronchospastic action via histamine release. In the present study, as acetaldehyde-induced bladder contraction was also completely blocked by the $\mathrm{H} 1$ antagonist, bladder contraction was probably enhanced by the release of histamine" $[27,28]$.

Bayrak $\mathrm{O}$ et al. writed, "To demonstrate the effects of intra-vescical ozone treatment on inflammation and epithelial cell damage in chemical cystitis animal model.

A total of 30 New Zealand rabbits were divided into 6 groups. Cystitis was conducted 
with a transurethral intra-vesical HCL- instillation on the subjects in Groups IA, IB, IIA, and IIB. Group IA-IB subjects was transurethrally administered intravesical ozonetherapy twice a week, Group IIA-IIB subjects were only given an intravesical isotonic $\mathrm{NaCl}$ instillation. Group IIIA-IIIB subjects were administered intravesical isotonic $\mathrm{NaCl}$ instillation without conducting chemical cystitis in order to create the same stress. Following a 3-week (early period) and 6-week (late period) therapy, the rabbits were sacrificed and histo-pathologic investigations tests were carried out in order to demonstrate changes in the urinary bladder.

We observed that the basal -membrane and the mucosal integrity were maintained, the inflammatory cells were suppressed in Group IA-IB (Early and late period), which received ozone therapy. However, it was observed that the mucosal integrity was spoiled, numerous inflammatory cells were accumulated in Group IIA-IIB, which was administered isotonic $\mathrm{NaCl}$.

Due to its low cost and minimal side effects; ozone therapy could be a new therapeutic approach in the treatment of interstitial cystitis" [29].

According Subhash Haldar et al., "Cystitis can be: acute or chronic, and severity can range from mild discomfort in the lower abdomen to severe life-threatening hemorrhage.

The many etiologies of cystitis: infection, radiation, chemical, mechanical, interstitial cystitis/chronic pelvic pain syndrome, and several conditions that can masquerade as cystitis. Cystitis can be classified: infectious versus non-infectious" [30].

Saeid Golbidi et al. writed, "Micturition is a highly-coordinated process that starts with sensing bladder filling, transferring data to the CNS system processing center and efferent pathways resulting in the voiding response. Increasing the volume of urinary bladder provokes afferent- signals to the central nervous system. The molecularbasis of distention sensing has not been fully clarified until today, may involve the urothelium as a major factor during this process. The urothelium represents the layer of epithelial cells lining the urinary tract between the renal pelvis and the urinary bladder. For years, it was considered like a passive barrier for passage of ions and solutes, currently is known to possess a number of features that allow it to respond to various physical / chemical stimuli, resulting in the release of various substances from the urothelial cells: these include ATP, nitric oxide (NO), substance P, acetylcholine, adenosine, antiproliferative factor, cytokines, various trophic factors, and prostanoids.

During bladder filling at low volumes, low frequency impulses activate afferent nerves to signal the pontine storage and micturition centers, and stimulate sympathetic outflow via hypogastric and pudendal nerves. Released norepinephrine induces the detrusor relaxation with enhances bladder compliance, which is mediated largely through $\beta_{3}$-adrenergic receptors, while bladder outlet resistance is enhanced by activation of $\alpha_{1}$-adrenergic receptors in the bladder neck, urethra and prostate. Somatic nerves also participate in the process of increasing bladder outlet resistance by releasing acetylcholine.

Increasing THE urine volume will increase frequency of afferent impulses, which in turn will shift efferent- signaling from the pontine storage to the micturition center. This activates parasympathetic and inhibit sympathetic system outflow. The resultant acetylcholine stimulates detrusor muscle through mainly $\mathrm{M}_{3}$ muscarinic receptor subtypes and also $\mathrm{M}_{2}$ recep. During pathophysiological situations.

The 2 most frequent causes of incontinence are excessive pressure development by the bladder (urgency urinary incontinency) and reduced bladder outlet resistance: stress urinary incontinence. An overactive bladder originates from involuntary detrusor- contractions during the filling phase of micturition cycle; it can occur with / without incontinence, and frequently manifests as urinary frequency and nocturia. 
Diabetic bladder dysfunction conditions presents in a spectrum of clinical symptoms representing various of disorders ranging from bladder overactivity to impaired bladder contractility. Since diabetes tends to occur in the elderly population, the concurrent factors such as benign prostatic hyperplasia, neurological disorders, and aging may also contribute to voiding dysfunction, makes it difficult to assess the specific contribution of the diabetic state to voiding dysfunction in these patients.

Diabetic cystopathy was used by F. Moller in 1976 and refers to lower urinary symptoms due to the diabetic neuropathy.

In Diabetic cystopathy: increased post voiding residual volumes and enhanced bladder capacity, decreased bladder sensation, contraction secondary to damage of visceral afferent fibers in the bladder wall.

This process causes gradual changes in patient's voiding patterns, with a reduced desire to void, whit stored urine volume of $350-400 \mathrm{ml}$ in healthy subjects.

This patients commonly experience difficulty in initiating and maintaining the micturition. Voiding- reflexes appear sluggish and an asymptomatic increase in bladder capacity and urinary retention occurs. Diabetic bladder dysfunction present as an overactive bladder syndrome usually, urgency, with / without incontinence, with urinary frequency and nocturia.

Indeed bladder hyper-sensitivity / hypercontractility is much more common than bladder hypo-contractility. More than half $(\sim 55 \%)$ of diabetic patients have detrusor hyperreflexia, another $23 \%$ have reduced detrusor contractility and a $10 \%$ demonstrate detrusor areflexia with the remaining $11 \%$ showing indeterminate findings. Based on both animal studies and human findings, Daneshgari et al., hyperglycemia-induced polyuria plays a major patho-physiological role during early stages of diabetes polyuria, causing compensatory bladder hypertrophy and associated myogenic and neurogenic alterations. This stage is compatible with the findings of a hyperactive bladder during urodynamic- evaluation when patients present with bladder storage concerns (urgency or urge incontinence). With time and accumulation of the toxic metabolites, decompensation of bladder- tissue and function ensues, resulting in the signs / symptoms of diabetic cystopathy (hypocontractile detrusor or atonic bladder) in patients with the urinary voiding problems" [31].

Victor W Nitti showed that in men and woman, "Primary bladder neck obstruction PBNO is a condition in which the bladder neck does not open appropriately or completely during voiding. Symptoms include: storage symptoms (frequency, urgency, urge incontinence, nocturia) and voiding symptoms (decreased force of stream, hesitancy, incomplete emptying). There are many theories as to the etiology of PBNO, including muscular / neurologic dysfunction and fibrosis. The diagnosis of PBNO can be made precisely with videourodynamics, urodynamic testing with simultaneous pressureflow measurement, and visualization of the bladder neck during voiding. Treatments: various from watchful waiting to medical therapy to surgery, depending on the severity of symptoms, urodynamic findings, and response to the described therapy.

In PBNO bladder neck fails to open adequately during voiding fases, with in increased striated sphincter activity or obstruction of urinary- flow in the absence of another anatomic obstruction, such as that caused by benign prostatic enlargement Bph in men or genitourinary prolapse in women. In 1973, Turner-Warwick et al introduced the use of urodynamics and voiding cystourethrography to diagnose bladder neck dysfunction in men. Norlen - Blaivas in 1986 diagnosed vesical neck obstruction in 23 young and middle-aged men with prior diagnoses of prostatitis, neurogenic bladder, and psychogenic voiding dysfunction. 
PBNO Etiology has not been clearly elucidated. First theories focused on structural changes at the bladder neck, such as a fibrous narrowing or hyperplasia, as initially proposed by Marion-Leadbetter proposed that there is a fault of dissolution of mesenchyme at the bladder neck or inclusion of abnormal amounts of nonmuscular connective tissue, resulting in hypertrophic smooth muscle, fibrous contractures, and inflammatory changes. Similarly, Turner-Warwick and colleagues described inefficient bladder neck opening resulting from abnormal morphologic arrangement of the detrusor/trigonal musculature.

A neurologic etiology in the form of sympathetic nervous syst. dysfunction has also been suggested. Crowe and coll. demonstrated an increase in the density of neuropeptide Y-immunoreactive nerves, part of the sympathetic contractile system of the bladder neck, in bladder neck tissue obtained from men with bladder neck dyssynergia.

Cases of apparent bladder neck dysfunction may actually be the result of abnormalities of the striated urethral sphincter. It is widely accepted that the $1^{\text {th }}$ event in volitional micturition is relaxation of the external striated sphincter. Yalla and Resnick showed that, as the external sphincter relaxes and pressure drops, the pressure in the bladder and at the bladder neck increases. The rate of increased pressure in the bladder is greater than that at the bladder neck.

When vesical pressure exceeds the vesical neck pressure, which occurs "within a few seconds," voiding ensues.In certain subjects, the periurethral striated muscle can extend functionally to the bladder neck.

In such patients, the pressure changes during the initiation of voiding may be slightly altered. In another study, Yalla and colleagues demonstrated that such extension of the functional external sphincter to the bladder neck occurred in $48 \%$ of men.

Watchful -waiting is an option for the patients who are not bothered much by their symptoms and have no clinical or urodynamic evidence of upper and/or lower urinary tract decompensation.

It is not known how many men or women who elect watchful waiting have progressive symptoms, develop decompensation, or subsequently receive treatment. It is likely that, in some cases, older men with LUTS presumed to be caused by BPH who had the start of symptoms in their twenties or thirties actually have long-standing PBNO.

$\alpha$-Blockers have been the mainstay of pharmacotherapy for PBNO. Although much of their effect is presumed to involve the smooth muscle of the bladder neck, $\alpha$-blockers may also affect the bladder via local or central mechanisms, as is assumed to be the case in BPH therapy. Unlike $\alpha$-blocker therapy for BPH, only variable success has been reported for $\alpha$-blocker therapy for PBNO.

In 1994, Kaplan and colleagues reported failure of $\alpha$-blockade in 31 men who ultimately underwent surgical intervention. For the patients who continued $\alpha$-blocker therapy, AUA symptom scores improved $67 \%$.

Nitti and colleagues reported that: 10 (58\%) of 17 men with PBNO had significant improvement in AUA symptom score with $\alpha$-blocker therapy;. In a study by Yang and colleagues, 24 men with PBNO received doxazosin, $1 \mathrm{mg}$ to $2 \mathrm{mg}$ daily. Fifty-eight percent of subjects had a reduction in International Prostate Symptom Score (IPSS) of greater than $50 \%$. The researchers also noted that men with positive responses had higher mean detrusor pressures at maximum flow and lower mean $Q_{\max }$ than did nonresponders. Treatment was more likely to be successful in patients who were urodynamically obstructed.

It appears that $\alpha$-bloc kers have a urodynamic effect on PBNO in men. 
There is 1 published study, by Kumar and colleagues: 24 women with PBNO received initial treatment with an $\alpha$-blocker. Subjects were highly symptomatic, and all with a significantly elevated PVR. PBNO was diagnosed using stringent urodynamic criteria. Fifty percent of subjects responded to $\alpha$-blockade with decreased symptoms, increased flow, and decreased PVR. Specifically, in responders, $Q_{\max }$ increased from 9.5 $\mathrm{mL} / \mathrm{s}$ to $15.1 \mathrm{~mL} / \mathrm{s}$ and PVR decreased from $277 \mathrm{~mL}$ to $27 \mathrm{~mL}$. No validated symptom assessment was used to evaluate symptom response.

PBNO can be treated surgically with unilateral /bilateral transurethral incision of the bladder neck.

Bladder neck incision is highly effective. In 1986, Norlen and Blaivas performed incision or resection in 18 of 23 men with PBNO. All patients experienced "symptomatic relief" (no objective parameters were used), and mean $Q_{\max }$ increased from $9.1 \mathrm{~mL} / \mathrm{s}$ to $26.1 \mathrm{~mL} / \mathrm{s}$.

In 1994, Kaplan and colleagues reported the results of unilateral incision in 31 men. That experienced a subjective improvement in symptoms, with mean Boyarsky symptom score decreasing from 16.4 to 6.4 and mean $Q_{\max }$ increasing from $9.2 \mathrm{~mL} / \mathrm{s}$ to $15.7 \mathrm{~mL} / \mathrm{s}$. No retrograde ejaculation was reported in this series with unilateral incision.

In a study by Trockman and colleagues, 18 of 36 men diagnosed with PBNO underwent bilateral incision. A "successful" outcome was obtained in 16 (89\%) of the men. Mean AUA symptom score decreased from 17.1 to 4.3 , and mean $Q_{\max }$ increased from $8.2 \mathrm{~mL} / \mathrm{s}$ to $26.7 \mathrm{~mL} / \mathrm{s}$. Antegrade ejaculation was maintained in $73 \%$ of subjects.

Bladder neck incision and resection may also be used to treat PBNO in women. Axelrod and Blaivas performed bilateral incisions at 5 o'clock and 7 o'clock in 3 women with PBNO and reported success in all cases, with no subject developing incontinence.

Gronbaek and colleagues performed a single incision initially and a second incision as needed in 38 women with PBNO. At a mean follow-up of 55 months, the success rate was $76 \%$. One patient (3\%) developed incontinence. Kumar and colleagues performed a single incision at 12 o'clock using a pediatric resectoscope in 6 women with PBNO who had failed $\alpha$-blockade. Success was reported in all 6 subjects, with $Q_{\max }$ increasing from $8.5 \mathrm{~mL} / \mathrm{s}$ to $15.5 \mathrm{~mL} / \mathrm{s}$ and PVR decreasing from $256 \mathrm{~mL}$ to $40 \mathrm{~mL}$. Mild stress urinary incontinence was reported in 2 (33\%) of the women" [32].

Chen $\mathrm{C}$ et al., "To explore the etiology of male urethral stricture, analyzing the therapeutic strategies of urethral stricture, and summarize the complicated cases.

183 patients data with urethral stricture were retrospectively analyze(, including etiology, obstruction site, stricture length, therapeutic strategy, and related complications). The mean age was abount 49.7 years, the average course was 64.7 months, and the constituent ratio of 51 to 65 years old patients was $38.8 \%(71 / 183)$. The traumatic injury of the patients accounted for about $52.4 \%$ (96/183), in which the pelvic fracture accounted for about $35.5 \%(65 / 183)$ and the straddle injury accounted for $16.9 \%$ (31/183). There were 54 cases of iatrogenic injury (about $29.5 \%)$. The posterior urethral stricture accounted for $45.9 \%(84 / 183)$, followed by the anterior urethral stricture $(44.8 \%, 82 / 183)$ and the stenosis $(6.6 \%, 12 / 183)$. A total of 99 patients (about $54.1 \%$ ) received the end to end anastomosis, 40 (21.9\%) were treated with intracavitary surgery, such as endoscopic holmium laser, cold knife incision, endoscopic electroknife scar removal, balloon dilation, and urethral dilation. In the patients $>65$-years old, the urethral stricture rate was: $14.8 \%$ and the complication rate $70.4 \%$ for transurethral- resection of the prostate (TURP) was significantly higher than that of all samples $(\mathrm{P}<0.01)$. The etiology of male urethral stricture and the treatment strategy have changed and the incidence of traumatic and iatrogenic urethral stricture has increased in the recent 3 years. The main treatment of urethralstricture has been transformed from an endoscopic surgery in urethra-plasty" [33]. 
Simon van Rij et al., "Management of BPH has often been divided into medical and surgical options. In 2015 the lines between the 2 are now more blurred. (This has been driven by patients' expectations of their treatments and new technology making the treatments less invasive). In the last time, no new drugs-medications that specifically act on the prostate have been brought to commercial market.

Alpha-blockers, which cause a relaxation of the smooth muscle fibers within the prostate, continue to be the 1th -line treatment. Recent research shows that only $40 \%$ of patients commenced on these drugs remain on treatment 6 months later. An alternative drugs are the 5-alpha reductase inhibitors. Large multi-centre randomised controlled trials have shown the benefit of these medications in improving urinary symptoms. However, evidence in relation to the side effects of these drugs has surfaced over the last few years. The most publicized came from the Prostate Cancer Prevention Trial, in which men on finasteride showed a possible increase in high-risk prostate cancer compared with those on placebo. The absolute difference in cancer rates was extremely low, and subsequent commentaries and analysis have aimed to disprove this. The other concerning side effect of finasteride has been a reported worsening sexual function, which in some men can be longstanding. The only new medications on the market for BPH in the last few years have been the phosphodiesterase type 5 (PDE5) inhibitors, most notably tadalafil. The indication for this medication is the treatment of erectile dysfunction, but trials have shown improvement in BPH symptoms without adverse sexual side effects. The mechanism for the effect of tadalafil for symptomatic BPH still has not been elucidated. It is possible that it has little effect on BPH but that because men have improved sexual function they feel better!" [34].

Rehan Nasir Khan et al. showed, "Normal urinary flow is a really complex and synchronized process, dependent by different factors: detrusor contractility, urethral resistance and both autonomic and voluntary nerve innervations, and may even be influenced by abdominal straining.

Micturation patterns have also proven to be affected by: age, gender, the neurological conditions, physiological state circadian rhythm.

A descriptive measurement of urinary flow rate and general voiding function is provided by uroflowmetry UFM. This provides not only specific measurements, but gives: graphical representation of the patient's stream, and an important role in diagnoses - management of various urological ailments for decades.

It may also be used as a rapid screening tool, alongside measurement of post-void residual (PVR) volume, for the effectiveness of voiding.

A total of 50 volunteers were inducted. In standing position the maximum flow rate was $32.4 \pm 9.6 \mathrm{ml} / \mathrm{sec}$, average flow rate $8.8 \pm 16.9 \mathrm{ml} / \mathrm{sec}$, and post-void residue was $8.8 \pm 16.9 \mathrm{ml}$. In sitting position, the maximum flow rate was $14.8 \pm 5.4 \mathrm{ml} / \mathrm{sec}$, average flow rate $14.8 \pm 5.4 \mathrm{ml} / \mathrm{sec}$, and post-void residue was $6.6 \pm 9.4 \mathrm{ml}$. A significant difference in both voiding positions was observed regarding the maximum flow rate, average flow rate $(\mathrm{p}=0.001 ; \mathrm{p}=0.003)$. The difference was not significant for voided volume $(p=0.676)$, or post-void residual volume $(p=0.771)$ in the either position. Agewise stratification of data showed significant difference in both younger and older age groups in terms of maximum flow rate $(p=00.19)$. (The average flow rate was only significantly different in younger age group) $(p=0.00)$. There was no difference in voided volume or post-void residue in either age group ( $p>0.05)$.

Our findings of lower urinary flow- rates in the sitting position can be better eXplained by a study conducted by Bockus et al.:

They reported that sitting position stretches the puborectalis muscle which slightly 
closes urogenital hiatus (thus causing slight obstruction at urogenital hiatus during micturition, at sitting position independent to presence / absence of any bladder outlet obstruction.) This relation can also be correlated anatomically, as described by Rad et al.

It was found that the average angle between rectum and the anal canal was about $92^{\circ}$ while sitting and becomes $132^{\circ}$ when the patient gets to squatting position. This change in angle may lead to the relaxation of puborectalis muscles leading to an easier bladder and bowel evacuation.

The standing voiding position in healthy people influenced uroflowmetry findings, and associated with higher flow rates" [35].

Gravas S et al., "To evaluate the effect of hexanic extract of Serenoa repens (HESr) on prostatic inflammation in patients with diagnosed prostatic inflammation.

Patients with prostatic inflammation histologically confirmed by TRUS prostatic biopsy were randomized either to receive HESr (320 mg/day) or no treatment.

A 2 nd biopsy was performed 6 months later according to standard clinical practice. Inflammation was assessed by the Irani's score and immune-histochemical staining using the CD3, CD4 and CD8 (for T-leucocytes), CD20 (for B-leucocytes) and CD163 (for macrophages) antibodies.

Overall 97 patients were eligible. In the HESr group the mean inflammation grading and aggressiveness grading score significantly decreased from 1.55 and 1.55 at baseline to $0.79(\mathrm{p}=0.001)$ and $0.87(\mathrm{p}=0.001)$ at the $2^{\text {nd }}$ biopsy, respectively. In the control group the mean inflammation grading score was 1.44 at first biopsy and 1.23 at the second biopsy. The mean aggressiveness gradings core was 1.09 and 0.89 , respectively. No statistical significance was found $(p=0.09$ and $p=0.74)$.The mean decrease in all inflammation scores was statistically higher in the HESr patients compared to controls. The immune-histochemical staining showed a significant change in the expression of the analyzed antibodies for the HESr patients compared to first biopsy. In the nontreatment group, no significant difference was found at the $2^{\text {nd }}$ biopsy. The change in expression of each antibody in the HESr group was statistical significant compared to control.

HESr seems to reduce prostatic inflammation in terms of histological and immunohistochemical parameters in this specific patients population" [36].

Morelli A et al., "In humans, prostate phosphodiesterase type 5 inhibitors (PDE5) expression was prominently localized in the endothelial and smooth muscle cells of the vascular bed, suggesting a possible action of PDE5 inhibitors (PDE5i) on prostate blood flow.

To investigate the PDE5 expression (human and rat) lower urinary tract tissues, including vasculature, and determine effects of PDE5 inhibition with tadalafil on the prostatic blood perfusion.

Human vesicular-deferential arteries (which originate from the inferior vesical artery, the main arterial source of blood supply to the bladder and prostate) were analyzed for PDE5 expression and activity. The effects of tadalafil on prostate -oxygenation were studied in spontaneously hypertensive rats (SHR), characterized by ischemia/hypoxia of the genito-urinary tract.

PDE5 expression was evaluated by quantitative reverse PCR and immunehistochemistry. SHR were treated with tadalafil (2 $\mathrm{mg} / \mathrm{kg} /$ day) for 1, 7, 28 days and compared with untreated SHR and the unaffected counterpart Wistar-Kyoto (WKY) rats. Prostate oxygenation was detected by Hypoxyprobe-1 and hypoxia markers (hypoxia-inducible factor-1 $\alpha$ [HIF-1 $\alpha]$ and endothelin-1 type B [ETB]) immunostaining. 
Human vesicular-deferential artery expressed high levels of PDE5, similar to corpora cavernosa, immunolocalized in the endothelial and smooth muscle layer. In these arteries, tadalafil inhibited cyclic guanosine monophosphate breakdown (half maximal inhibitory concentration in the low nanomolar range, as in corpora cavernosa) and increased the relaxant response to sodium nitroprusside. SHR prostate resulted markedly hypoxic (hypoxyprobe immunopositivity) and + for HIF-1 $\alpha$ and ETB, while tadalafil treatment restored oxygenation to WKY level at each time point. The mRNA expression of the HIF- $1 \alpha$ target gene, BCL2/adenovirus E1B $19 \mathrm{kDa}$ interacting protein 3, significantly increased in SHR prostate and partially restored to WKY level by tadalafil. Human vesicular-deferential artery present high expression and activity of PDE5, which was inhibited by tadalafil in vitro. In SHR, tadalafil increases prostate tissue oxygenation, a possible mechanism through which PDE5i exert beneficial effects on LUT symptoms" [37].

Fibbi B et al., "PDE5 is highly expressed in rat and human bladder, where it regulates cyclic guanosine monophosphate (cGMP) degradation, muscle tone, and proliferation.

PDE5 expression were analyzed and compared within the same BPH patient in LUT tissues and in smooth muscle cells (SMCs) cultured from urethra, prostate, and bladder.

In LUT tissues, PDE5 was localized by immune-histochemistry and mRNA expression by quantitative real-time PCR. Proliferation assay was used as readout of PDE5 activity, evaluated as ability of vardenafil to increase the antiproliferative effect of different nitric oxide (NO)/cGMP pathway activators [the PDE5-resistant cGMP analog Sp-8-Br-PET-cGMPS, the NO donor sodium nitroprusside (SNP), and the soluble guanylate cyclase (sGC) stimulator BAY 41-8543].

In all the LUT tissues, PDE5 was immune-localized in blood vessels and in muscular fibres, but not in epithelium. PDE5 mRNA expression was higher in urethra and bladder than in prostate SMC. The antiproliferative effect of Sp-8-Br-PET-cGMPS was similar in all LUT SMC. In prostatic SMC, SNP and BAY 41-8543 show a dose-dependent antiproliferative effect that resulted marginally enhanced by vardenafil. Conversely, in urethra and bladder SMC the antiproliferative effect of SNP and BAY 41-8543 was lower than in prostatic SMC, it was significantly enhanced by vardenafil treatement. In urethral- bladder cells vardenafil half-maximal response inhibiting concentration was in the sub-nanomolar range, whereas in prostate cells it resulted significantly higher.

The highest expression and biological activity of PDE5 was found in bladder. A consistent PDE5 expression and activity was found in prostatic urethra. In contrast, the prostate gland showed the lowest PDE5 abundance and cultures derived from this tissue were less sensitive to vardenafil" [38].

Krivoborodov GG et al., The efficacy of treatment of chronic pelvic pain syndrome in combination with bladder emptying dysfunction was comparatively analysed in 31 male patients. Statistics show that current schemes of medication including antibacterial, nonsteroid anti-inflammatory drugs, alpha-adrenoblockers are not highly effective in such patients. Trans-rectal microwave hyperthermia is effective in males with chronic pelvic pain syndrome but the response is short - 3 months maximum. The highest and longest effect in patients with chronic pelvic pain syndrome in combination with bladder emptying dysfunction was achieved after injections of 100 units of botulinic toxin type A into the cross-striated sphincter of the urethra [39].

Zhanel GG et al., "Acute - chronic bacterial prostatitis in outpatients is commonly treated with oral fluoro-quinolones; but the worldwide dissemination of multidrug-resistant (MDR) Escherichia coli has resulted in therapeutic failures with fluoroquinolones. We reviewed the literature regarding the use of oral fosfomycin 
in the treatment of acute and chronic prostatitis caused by MDR E. coli. All Englishlanguage references on PubMed from 1986 to June 2017, inclusive, were reviewed from the search "fosfomycin prostatitis."

Fosfomycin demonstrates potent in vitro activity against a variety of antimicrobialresistant $E$. coli genotypes/phenotypes including ciprofloxacin resistant, trimethoprim -sulfamethoxazole-resistant, extended-spectrum $\beta$-lactamase- (ESBL-) producing, and MDR isolates. Fosfomycin attains therapeutic concentrations $(\geq 4 \mu \mathrm{g} / \mathrm{g})$ in un-inflamed prostatic tissue and maintains a high prostate/plasma ratio up to 17 hours after oral administration. Oral fosfomycin's clinical cure rates in the treatment of bacterial prostatitis caused by antimicrobial-resistant E. coli ranged from 50 to $77 \%$ with microbiological eradication rates of $>50 \%$. Oral regimen of fosfomycin tromethamine of $3 \mathrm{~g} \cdot \mathrm{q} 24 \mathrm{~h}$ for one week followed by $3 \mathrm{~g} \cdot \mathrm{q} 48 \mathrm{~h}$ for a total treatment duration of 6-12 weeks appeared to be effective. The Oral fosfomycin may represent an efficacious and safe treatment for acute and chronic prostatitis caused by MDR E. coli “[40].

Carsten Niemitz, "During the last century, more than 30 hypotheses have been constructed to explain the evolution of the human upright posture and locomotion. It has been established that all main hypotheses published until the last decade of the past century are outdated, at least with respect to some of their main ideas: Firstly, they were focused on only one cause for the evolution of bipedality, whereas the evolutionary process was much more complex. Second, they were all placed into a savannah scenario. During the 1990s, the fossil record allowed the reconstruction of emerging bipedalism more precisely in a forested habitat. The fossil remains revealed increasing evidence that this part of human evolution took place in a more humid environment than previously assumed. The Amphibian Generalist Theory, presented first in the year 2000, suggests that bipedalism began in a wooded habitat. The forests were not far from a shore, where our early ancestor, along with its arboreal habits, walked and waded in shallow water finding rich food with little investment. In contrast to all other theories, wading behaviour not only triggers an upright posture, but also forces the individual to maintain this position and to walk bipedally. So far, this is the only scenario suitable to overcome the considerable anatomical and functional threshold from quadrupedalism to bipedalism. This is consistent with paleo-anthropological findings and with functional anatomy as well as with energetic calculations, and not least, with evolutionary psychology. The new synthesis presented here is able to harmonise many of the hitherto competing theories" [41].

According Whi-An Kwo, "Interstitial cystitis/bladder pain syndrome (IC/BPS) is a chronic / heterogeneous syndrome characterized by long periods of bladder, pelvic, or perineal pain, urinary frequency, nocturia, and urinary urgency. IC/BPS seriously impairs the global quality of life and is a significant burden on patients. Most issues related to IC/BPS, including definition/ incidenc/ etiology/ pathology, have been controversial, and its diagnosis and definition are constantly changing. There is also no known treatment for IC/BPS, and the pathophysiology of this disease is still poorly understood.

The proposed etiology includes inflammatory, autoimmune, neurotoxic, and vascular components. Disappearance of the glycosaminoglycan layer from superficial urothelial cells and urinary toxicity have been proposed as pathophysiological mechanisms.

Studies have been performed on the therapeutic use of stem cells for IC/BPS treatment, and many other in vivo studies are underway. In past decades, many models have been developed, with characteristics similar to those of the IC phenotype. Most animal models have been produced by injection of: chemical toxins or stimulants into the bladder or by systemic injection of chemical agents, viruses, or antigens that cause inflammation of bladder. 
This review noted that it is not possible to effectively characterize IC/BPS in humans using a single model, and research has recently focused on models that are widely used to reflect the characteristics of IC/BPS patients. These models are classified into 3 categories: (1) bladder-centric models, (2) models with complex mechanisms, and (3) psychological and physical stressors/natural models.

If we cannot identify the pathophysiology and course of IC/BPS, we will not be able to meet the targets of therapy" [42].

Avril Lusty et al. showed that, "1628 adult females with a self-reported diagnosis of IC completed a web-based survey in which patients described their perceived outcomes with the therapies they were exposed to. Patient-reported outcomes were compared to available clinical trial outcomes and published treatment guidelines.

Based on patient perceived outcomes (benefit: risk ratio), the most effective treatments were: opioids, phenazopyridine, and alkalizing agents, with amitriptyline and antihistamines reported as moderately effective. The only surgical procedure with any effectiveness was electrocautery of Hunner's lesions. In order of efficacy reported in the literature, the therapies for IC/BPS with predicted superior outcomes should be: cyclosporine- A, amitriptyline, hyperbaric oxygen, pentosan polysulfate plus subcutaneous heparin, botulinum toxin A plus hydrodistension, and L-arginine" [43].

"Therapies achieving an improvement rate of over 50\% included opioids (405/621; $65.2 \%)$, phenazopyridine $(390 / 638 ; 61.1 \%)$, intravesical heparin $(50 / 89 ; 56.2 \%)$, alkalinizing agents $(365 / 660 ; 55.3 \%)$, and pentosan polysulfate $(359 / 698 ; 51.4 \%)$; however, in order of effectiveness (based on benefit: risk ratio), marked effectiveness was only achieved with opioids (27:1), alkalinizing agents (22.8:1), phenazopyridine (13.9:1). A Moderate effectiveness was reported for amitriptyline (9.2:1) and the antihistamines (6.5:1). Cimetidine, intravesical heparin, and intravesical hyaluronic acid were associated with mild effectiveness, while electrocautery, pentosan polysulfate, intravesical lidocaine, dimethylsulfoxide DMSO showed mild but equivocal effectiveness. Hydrodistension and antibiotics were not effective (although antibiotics were associated with a $49.4 \%$ improvement rate, they also had worsening of symptoms, reported by $47.1 \%$ of patients). Gabapentinoids, major surgery, and urethral dilation were not effective" [43].

Kristina Cvach et al., "Bladder pain syndrome/interstitial cystitis (BPS/IC) is a chronic -pain condition whit urinary frequency/ urgency and pain o/ discomfort which the patient attributes to the bladder. It is a complex situations to manage and treat and requires a multi-disciplinary and multi-modal approach. As well as lifestyle and behavioural modifications, physical therapy and oral medications, intravesical treatments can be used in the treatment algorithm for BPS/IC.

According to the American Urological Association guidelines, symptoms should be present for a period of more than 6 weeks. Confusable diseases, such as overactive bladder and recurrent urinary tract infection, need to be excluded prior to a diagnosis of BPS/IC.

The urothelium/transitional epithelium is thought to play a great role in the pathogenesis of BPS/IC. The protective layer of glycosaminoglycans (GAG) on the surface of the urothelial cells provides a barrier against solutes in the urine. Components of layer include hyaluronic acid (HA), chondroitin sulfate (CS), heparin sulfate, dermatan sulfate and keratin sulfate. The GAG layer has been shown to be defective in some patients with BPS/IC.

Once the subepithelial cells come into contact with urinary solutes, an inflammatory reaction is triggered, resulting in decreased urothelial production of GAG and 
exacerbation of urothelial permeability / inflammation. Urothelial damage results in pathologic C-fiber activation, this cause smooth muscle contraction, the neurogenic inflammation, and global hypersensitivity. As with other chronic pain conditions, a central pain sensitisation results.

First line therapy is aimed at educating the patient about the chronic nature of the condition and advocating behavioural - lifestyle modifications that may help and ameliorate the global symptoms, as well as providing a pain management plan.

Intravesical therapies are indicated if first line therapy failed and are used in conjunction with the physiotherapy techniques (myofascial release/pelvic floor muscle relaxation) and the oral agents. (to replenish the deficient GAG layer or to alter the process of neurogenic inflammation - hypersensitivity.)

Intravesical therapy has the advantages of localize therapy into the bladder, with the establishment of high concentrations of the drugs and agent and minimising systemic side effects. The disadvantages are that delivery of the agent requires instrumentation of the urethra and bladder with the potential for exacerbating of pain and increasing the risk of urinary tract infection.

\section{Intravesical therapies}

Dimethyl sulfoxide DMSO is an organo-sulfur non-toxic solvent, used in BPS/IC since the 1970's when it was approved by the US FDA for intra-vesical use. It is likely to exert it's the clinical effect through many mechanisms: reducing inflammation, acting as an analgesic and facilitating detrusor relaxation. It can be given as: singleagent instillation at a $50 \%$ concentration or, in a 'cocktail' with methylprednisolone or hydrocortisone, alkalised lidocaine and heparin sulfate. The Instillation- regimens differ but a common treatment program involves twice weekly instillations for 4 weeks, then weekly instillations for a further 4 weeks, for 12 instillations. Repeat full, partial (4 weekly instillations) or maintenance (monthly) treatment courses for persistent or recurrent symptoms are possible.

Response rates in these studies range from about 70-95\%.

Perez-Marrero et al. performed randomized-placebo-controlled crossover clinical trial in about 33 patients and assessment based on urodynamics and symptoms. Compared to the placebo, DMSO showed a higher objective (35\% vs. 93\%) and subjective (18\% vs. $53 \%)$ improvement.

Peeker et al., performed a randomized -double-blind crossover clinical study comparing DMSO to intravesical BCG in 21 patients. Each group underwent 6 weekly instillations and crossed-over to other treatment if no improvement was noted. The Patients were assessed with a symptom questionnaires (VAS pain scale and voiding diaries.) No improvement in any of the outcome measures were seen following BCG: the study was likely to be underpowered. DMSO resulted in significant reductions in pain and the urinary frequency parameter.

Ghoniem et al. utilised a DMSO cocktail: premixed $50 \mathrm{~mL}$ solution of $50 \%$ DMSO, $40 \mathrm{mg}$ methylprednisolone and 5,000 units of heparin sulfate in 25 patients. 6 instillations (the first performed under general anesthesia after hydrodistention) were administered at weekly- intervals. Outcome measures were not well defined, with $92 \%$ achieving an initial remission for an average of 8 months. 9 patients (36\%) had one or more relapses and required further instillations or oral therapy.

Lim et al. reported long-term outcomes following a DMSO cocktail- instillation (50 mL of 50\% DMSO, $100 \mathrm{mg}$ hydrocortisone, $0.25 \%$ bupivicaine and 5,000 units of heparin). Mean follow-up of about 55 patients was at 4.65 years and the overall 
improvement as assessed on ICSI/ICPI and VAS a pain scores was : 23-47\%. At longterm follow-up $34 \%$ were cured after 1 course, $22 \%$ required oral medication, $24 \%$ had a DMSO top-up; the remainder went on to receive the other treatments. Anesthetic bladder capacity about $\leq 500 \mathrm{~mL}$ was associated to a lower response rate.

Heparin and lidocaine: Heparin is a sulfonated- GAG with theoretical action of replenishing urothelial GAG layer. Heparin acts as anti-inflammatory molecule, inhibits the fibroblast proliferation and promotes angiogenesis and the smooth muscle cell proliferation. Lidocaine is a topic anaesthetic- drug and is used as a single-agent instillation or, in combination with the heparin. It is given in various formulations / concentrations, usually in combination with alkalizing- agent (sodium bicarbonate) to avoid the ionisation within urine andin order to better penetrate the urothelium.

Single-agent heparin studies have shown modest benefit in patients with BPS/IC. Parsons et al. reported on about 48 patients undergoing instillation with $10,000 \mathrm{U}$ of heparin 3 times a week for 3 months. $56 \%$ attained a good clinical remission.

Single alkalised lidocaine (200 mg lidocaine plus 8.4\% sodium bicarbonate, 10 $\mathrm{mL}$ ) was reported by the author Nickel et al., in a randomized, double-blind placebocontrolled industry sponsored study of about 102 patients. Instillations occurred daily for about 5- consecutive days. The primary clinical outcome was based on the Global Response Assessment. On day eight, patients in lidocaine group had significantly higher improvement than the placebo ( $30 \%$ vs. $10 \%, \mathrm{P}=0.012)$; this improvement was still present at day 15 (but not statistically significant 24\% vs. 12\%, P=0.102). 82 patients elected to continue the open-label phase of the clinical study and underwent a further 5-day course of the lidocaine treatement with similar rates of the improvement.

To improve the response rates, multi-agent therapy with: heparin and alkalised lidocaine has been studied. Parsons et al. have studied the efficacy of heparin and alkalised lidocaine in about 82 patients. Group 1 received about 40,000 units of heparin, $8 \mathrm{~mL}$ of $1 \%$ lidocaine and $3 \mathrm{~mL}$ of $8.4 \%$ sodium bicarbonate. Group 2 received increased concentration of lidocaine ( $8 \mathrm{~mL}$ of $2 \%$ lidocaine). An Immediate relief of the pain and frequency after 1 instillation was higher in group 2 than the group 1 (94\% vs. 75\%; $\mathrm{P}<0.01$ ). 20 patients in group 2 underwent a further 6 instillations over 2 weeks, $80 \%$ showed: symptom relief persisting at least for 48 hours after last treatment. The Improved duration effect was showed by Nomiya et al., in a clinical study of 32 patients undergoing 12 weekly instillations of 20,000 units heparin, $5 \mathrm{~mL} 4 \%$ lidocaine and 25 $\mathrm{mL} 7 \%$ sodium bicarbonate. On GRA, 76\% responded at the end of treatment, responses of $90 \%$ at 1 month, $46 \%$ at 2 months and $16 \%$ at 6 months- following the treatment.

\section{Pentosan Polysulfate Sodium (PPS)}

PPS is the only oral -agent approved for BPS/IC by FDA org. It is an oral -eparinoid molecule and likely exerts its effect for treatment of BPS/IC by restoring the GAG layer. It inhibits histamine release by the mast cells and reduces the intracellular calciumion levels in the bladder. Main disadvantage is the low urine concentrations achieved, resulting in a lag -time of up to the 6 months before clinical improvement is observed. Intravesical therapy has theoretical advantage of achieving more rapid response to PPS treatment.

Davis et al., randomised, double-blind controlled study comparing oral PPS and intravesical PPS to oral- PPS and intravesical placebo in about 41 patients. Instillations occurred 2 times weekly for 6 weeks whilst oral- therapy was given for a total of 18 weeks. At the end of the study period, the treatment group were shown to have a statistically greater reduction in ICSI/ICPI scores compared to placebo (46\% reduction vs. $24 \%$ reduction; $\mathrm{P}=0.04$ ). Health-related quality of life-domains also showed a statistically greater improvement in the treatment group. 
Sodium hyaluronate (HA) rationale is to replenish deficiencies in the GAG- layer (other biological activities including enhancement of connective tissue healing and inhibition of the leukocyte migration and aggregation may contribute to its action in BPS/IC patients. It comes as a $40 \mathrm{mg}$ dose in $50 \mathrm{~mL}$ solution). Most studies use a treatment regimen consisting of 4 weekly instillations followed by monthly- therapy until the symptoms have been resolved.

Riedl et al., prospectively studied the efficacy of HA in 126 patients as first line therapy. Weekly instillations of HA were given until the patients were significantly clinically improved or symptom free. The Assessment was based on a non-standardised questionnaire (using a VAS symptom- score, impact on quality of life question and willingness to undergo repeat the instillation therapy). This was administered at baseline and at a mean of 6 months after the last HA- instillation. The average number of instillations was 12.2.

About $85 \%$ of patients reported symptom improvement ( $\geq 2$ VAS units); $55 \%$ had no or minimal bladder symptoms after the therapy. 84\% reported an before rest improved quality of life (although this was not objectively quantified) and 86\% would undergo repeat HA treatment if necessary. 34\% had a recurrence of symptoms and required further instillations - the duration of the effect before a restarting treatment was not stated. The same group then published long-term follow-up data of: 48/70 patients from this cohort who were contacted at a mean follow-up of 5 years.

$50 \%$ had sustained improvement and did not require any further treatment. $41 \%$ continued with an intermittent HA instillation therapy with or without oral pentosan polysulfate.

A smaller case- series have reported an improvement rates with HA instillation of between: $30-85 \%$ with varying the follow-up times and instruments used to assess outcomes.

Shao et al., in an prospective open-label controlled clinical trial in 47 patients examining the use of HA after hydro-distention. Patients with functional bladdercapacities $<200 \mathrm{~mL}$ underwent hydrodistension followed by a treatment with either HA $(n=20)$ or a heparin/lidocaine cocktail $(n=16)$. Treatments occurred weekly for 4 weeks, then monthly for a further 2 months. 7 patients served as 'controls' undergoing only hydrodistention. Assessment was based on urinary frequency, bladder capacity, and VAS pain- score at baseline and 3, 6 and 9 months following hydro-distension. At 6 months, 78\% in the HA- group and 33\% in the heparin group had improvement in their symptoms vs $9 \%$ in the hydrodistension group. At 9 months, improvement was sustained (but diminished) in the HA group but not in the heparin group. There were no adverse- effects related to HA or heparin however two patients had bladderrupture during hydro-distension.

Gulpinar et al., reported the use of electromotive drug administration (EMDA) to improve the efficacy of HA treatment. EMDA increases the tissue uptake of drug compared with a passive diffusion. 31 patients were randomized to instillation of the HA alone $(n=15)$ or HA with EMDA with placement of suprapubic electrodes $(n=16)$. Patients were not blinded to the treatment allocation. Follow-up was at 1, 6, 12, 24 months. Both groups had statistically- significant improvement in all the parameters assessed (urinary frequency, nocturia, VAS- pain, voided- volume, ICSI/ICPI and global response assessment) at 6 - 12 months; the percentage of responders was higher in the EMDA group than in HA alone group (69\% vs. 58\%, P=0.042). By 24 months, only voiding frequency and VAS- pain scores were still significantly improved from the baseline in both groups.

2 industry-sponsored randomised, placebo-controlled clinical trials of HA were completed in 2003/04. Both trials showed a non-significant improvements in the treatment group compared to placebo and remain unpublished in the peer-reviewed literature. 


\section{Chondroitin Sulfate (CS)}

Is a component of the GAG layer and has been shown to be deficient in patients with BPS/IC. As its role in the GAG layer, CS inhibit the recruitment of inflammatory cells to the deep layers of bladder wall.

Nordling et al., conducted a multi-centre, prospective observe. Trial of $40 \mathrm{~mL}$ of $0.2 \%$ CS in 286 patients with chronic -cystitis (51\% were BPS/IC patients). Instillations occurred weekly for the first 4-6 weeks, then monthly until 12 weeks (with a maximum of 8 instillations). Outcome measures from baseline to end of study included a change in daytime and nocturnal frequency, urgency and pain- scores and a global response assessment. Overall, statistically significant changes were seen in all parameters and this was also seen in a subgroup analysis of the BPS/IC patients. GRA response was 76\%.

Nickel et al., multi-centre, prospective observational -study of $20 \mathrm{~mL} \mathrm{2 \%} \mathrm{CS.} 53$ patients underwent 6 weekly instillations followed by a further 4 instillations at monthly intervals. Global response was assessed at week 10, 24 (with rates of $47 \%$ and $60 \%$. At week 24 , ICSI and ICPI scores significantly decreased as did VAS pain and urgency scores, randomized, double-blind, inactive- vehicle control clinical trial of $20 \mathrm{~mL} \mathrm{2 \%} \mathrm{CS.} 98$ patients underwent eight weekly instillations, then monthly treatments to 10 . No statistically significantly results were obtained with respect to the GRA (treatment group 38\% vs. the control 31\%) or based on changes in ICSI/ ICPI scores/frequency/ urgency/ voided volume or VAS- pain scores. The study was under-powered and based on these results (would require a total of 1,500 patients to be adequately powered to show a difference in outcomes between the 2 groups), the author's conclusion was that single-agent therapy with CS could not be supported.

\section{Sodium Ayaluronate and Chondroitin Sulfate (HA-CS)}

Reduce the production of pro-inflammatory cytokines, reduce the urothelial permeability, facilitate the repair of protective GAG- layer. HA-CS is available as a 50 $\mathrm{mL}$ preparation containing 1.6\% HA and 2\% CS with calcium chloride in $\mathrm{H} 20$.

3 small case series with 63 patients have been published assessing treatment response to HA-CS. Instillations occurred weekly for a period of 4-20 weeks, further biweekly / monthly instillations until treatment response was assessed. All studies reported significant changes from baseline in ICSI/ICPI scores. Cervigni et al. showed significant changes from baseline in VAS pain - urgency scores.

Cervigni et al., presented at ICS 2014 an IBSA funded study comparing 13 weekly instillations of Ialuril with DMSO in a 2:1 random- allocation with 110 subjects with ESSIC criteria for BPS. The baseline demographics, VAS- pain scores were similar. The results were: VAS score reduction of -39 (SD 25) vs. -31 (SD 26) points for ialuril, DMSO respectively. Response at 6 months defined as a $>30 \%$ reduction of the VAS from the baseline was $73 \%$ vs. $58 \%$ for ialuril and DMSO respectively. Adverse- events were higher in the DMSO group at $31 \%$ vs. $15 \%$. There were 7 dropouts overall and were evenly divided.

\section{Oxychlorosene Sodium (OS)}

Is a stabilised organic- derivative of hypochlorous -acid. It has been used as antibacterial extensively in general surgery, particularly in the irrigation of wounds. Its use in the BPS/IC population stemmed from its role in TBC cystitis, when infection was thought to be an inciting event in the BPS/IC cascade. The mechanism of action of OS in BPS/IC is unclear but some have hypothesised that it may act by desensitising or degranulating bladder nociceptive nerve endings.

Initial cases published in the $1950-70$ 's provided some encouraging results, with response rates of $70-80 \%$. OS has been designated as a historical treatment in various 
reviews of intra-vesical therapy for BPS/IC. The authors are involved in a multi-centre, randomized, single-blind controlled clinical- trial assessing the efficacy of a singleinstillation $0.4 \%$ OS under general anesthesia compared to hydro-distension.

Botulinum toxin A potent neurotoxin, inhibit the release of acetylcholine and other neurotransmitters from both afferent and efferent nerve terminals as well as ATP from the urothelium. Chronic inflammation, apoptotic- signaling properties is significantly reduced following intra-vesical botulinum toxin A injections, but only after repeated injections. The recently amended AUA- guidelines for the treatment of BPS/IC have moved botulinum toxin A from 5 th to first 4 th line therapeutic treatment.

Most clinical studies of botulinum toxin a use onabotulinum toxin A. A number of case series with 11-16 patients each, showed that intravesical injections of 100-200 units of Botox resulted in high response rates 71-100\%. Significant reductions in VASpain scores, urinary daytime, nighttime frequency were seen at 3 months follow-up. These clinical studies varied in the dose injected, the number of injections and the site of injection: trigonal vs. supratrigonal.

Kuo et al. performed a multi-centre, randomised, double-blind placebo-controlled clinical- trial of Botox in BPS/IC patients refractory to the conventional treatment. 40 patients underwent suburothelial injections of 100 units Botox and hydrodistension whilst 20 contro-l patients underwent a normal saline injections and hydrodistension. At 8 weeks post-treatment, overall success was greater in Botox group compared to the control group ( $63 \%$ vs. $15 \%, \mathrm{P}=0.028)$. VAS pain scores $(-2.6$ vs. $-0.9, \mathrm{P}=0.021)$ and cysto-metric capacity ( +67.8 vs. $-45.4, \mathrm{P}=0.02$ ) were significantly changed in the Botox group compared to placebo. Adverse- events: dysuria (16/40 Botox, 1/20 control), urinary tract infection (2 Botox), urinary retention (1 Botox) and haematuria (1 Botox) were all greater in Botox group. In a prospective series of 44 patients undergoing 6 monthly Botox injections, Lee and Kuo reported that Botox was not beneficial in ulcer type BPS/IC patients.

Manning et al. reported about the use of botulinum toxin A. This was a multi-centre, randomised, double-blind placebo-controlled clinical trial in 54 patients. Dysport 500 U suburothelial injections plus hydrodistension was compared to a normal saline SOL. injections plus hydrodistention. O'Leary Sant overall scores were improved in the both groups at 3 months with no statistically significantly difference seen between the 2 groups. Only the OLS-problem index improved in Dysport group ( $\mathrm{P}=0.04)$. This study found that post-treatment UTI was a confounder and that when the 12 patients were excluded from the analysis, the OLS overall score and OLS-symptom index were significantly improved in the Dysport group. The authors concluded: Dysport may be beneficial in a small number of patients and that patients without post-treatment UTI presented a better response.

Capsaicin and the resiniferatoxin are C-fiber afferent neurotoxins. Considered as candidates for BPS/IC based on their theoretical ability to alleviate bladder symptoms by desensitising bladder afferents. Whilst the clinical- efficacy for resiniferatoxin was initially demonstrated in a number of small clinical studies, these results have not been confirmed in placebo-controlled clinical studies. They are currently not-recommended for intravesical use in BPS/IC patients.

Bacillus Calmette-Guerin (BCG), an immune-modulatory agent used for intravesical treatment of bladder cancer. An initial open label study provided promising results, BUT a randomized double blind study demonstrated no clinical benefit. Adverse events may be serious, so BCG is not recommended for use in BPS/IC patients.

Liposomes are phospholipid vesicles, when applied to cell -walls they create a molecular film. They may be able to restore the GAG layer. A small pilot -study has 
shown a significant improvement in frequency, nocturia, and pain in 24 patients undergoing intra-vesical liposomal instillations compared to oral PPS. A randomised, placebo-controlled clinical trial is currently underway assessing the efficacy of two doses of intravesical liposomal therapy compared to placebo [44].

Sudhindra Jayasimha, "Nanotechnology has revolutionized our approach to the medical diagnostics / therapeutics and has spanned an entirely new branch of science research. This review studies addresses the potential applications of the Nanotechnology in Urology filed.

The developments in urology- diagnostics include novel techniques of imaging of genitourinary malignancies, prostate-specific antigen measurement, early detection of mutations that are diagnostic for polycystic kidney disease. The potential- applications of nanotechnology are in targeted therapy of genit-ourinary malignancies, erectile dysfunction, overactive bladder, bladder reconstruction, construction of artificial kidneys and biodegradable stents, in robotic- surgery.

\section{Treatment of overactive bladder/interstitial cystitis}

Intravesical therapy, while overcoming side effects of anticholinergics such as dry mouth and constipation, provides higher local drug concentrations / efficacy. Systemic toxicity is limited as the urothelium of the bladder is the most impermeable epithelialbarrier of the body. Weekly intravesical empty liposomes (80 $\mathrm{mg}$ in $40 \mathrm{ml}$ water) have been shown to ameliorate the symptoms of interstitial- cystitis with decreased frequency, nocturia, and 0'Leary-Sant symptom score. In this aspect, liposome therapy was found to be superior to oral pentosan poly-sulfate.

Liposomes may also be used as a vector for the botulinum neurotoxin. This improves the efficacy of the drug as uptake is better, the degradation by proteases is prevented. The need for an intravesical injections can be circumvented as the efficacy remains the same which was demonstrated in acetic acid rat- model. Imaging of interstitial cystitis is also aided by nano-technology as liposomes and fluorescent nano-particles bind to bladder lesions of interstitial cystitis which may be assessed by NIR-imaging of bladder" [45].

Martino Maria Zacchè, et al., "Novel targeted DDSs was recently developed to prolong the effect of drugs in bladder. This has been possible with introduction of nano-technology in the field of drug -delivery. Substances are loaded within nanoparticles. This holds a high potential in improving the therapeutic value of different drugs- MOLECULES by increasing bioavailability, solubility, and retention time.

Nano-carriers can deliver drugs molecule to site-specific targets. They can be formulated from materials like lipids, polymers, proteins, and metals.

Liposomes are phospholipid concentric /bilayer nano-spheres mimicking human cell walls. They allow the delivery of both the lipophilic drugs molecules such as capsaicin and hydrophilic drugs such as botulinum toxin. Clathrin-mediated endocytosis (is their route of entry into the urothelium).

Intra-vesical administration of the liposomes alone can enhance resistance to irritant penetration as shown in rat- model with hyperactive- bladder. Clinical Studies showed that intravesical liposomes are safe and have similar efficacy to one of the most effective FDA-approved pharmacological treatments for IC as an oral pentosan polysulfate (PP) (Empty liposomes have been shown to be effective as potential treatment of IC). This related to their ability to form a protective- lipid film on urothelial surface. In a study, intravesical instillation of PP encapsulated in liposomes was effective in refractory IC pat. Studies with bigger number and placebo groups are warranted. 
Liposomal- vesicles enhance the therapeutic index of chemo-therapeutic agents and represent a promising approach for cancer treatment. A Phase II clinical trial STUDY is evaluating temperature-sensitive liposomes plus local hyper-thermia in patients with recurrent chest wall breast cancer. Encouraging results could lead to explore this approach in different clinical setting like BC.

Other nano-carriers are solid lipid nanoparticles, protein nanoparticles with targeted ligands grafted on the surface, branched polymeric den-drimers, mucoadhesive bio-polymers (chitosan) or synthetic polymers, magnetic particles, gold nano-shells, and in situ gelling systems.

Polymeric hydrogels: a Novel approach is the use of hydrogels as depot formulations on the bladder- walls. This provides longer exposure of the urinary tract tissue to existing drugs, as compared to standard intravesical instillation, as they remain attached to the bladder wall even after urine voiding- fases.

Thermosensitive hydrogels: These are aqueous solutions of poly (ethylene glycol-b-[dl-lactic acid-co-glycolic acid]-b-ethyleneglycol) triblock co-polymers that form a free-flowing solution at room temperature and become a viscous gel at body temperature of $37^{\circ} \mathrm{C}$. (It is fluid when cold and a gel at body temperature). Following the instillation, gel solidifies and acts as sustained drug release depot in situ. When the gel is in contact with urine, it dissolves and gradually releases the drug over a period of $6 / 8$ hours. Thigel slowly excreted from the bladder during urination. It is $100 \%$ biocompatible and harmless to the body.

A Phase I/II clinical trial was completed in Israel exploring the feasibilitytolerability- safety of 200 units of onabotulinumtoxin A premixed with chilled $40 \mathrm{~mL}$ of TCGel®. Fifteen patients with IC were enrolled in the clinical trial and received a single instillation. Follow-up was at weeks 2, 6, 12 post-instillation. The efficacy outcome measures included IC- Symptom Index (ICSI) and IC Problem Index (ICPI). A reduction in the ICSI and ICPI was observed since week 2 and was also apparent at week 12 . No serious drug-related adverse events were reported.

- Clinical trials are looking to the intravesical instillation of mitomycin C (MMC) mixed with TCGel® in patients with non-muscle-invasive BC (Optimized Instillation of Mitomycin for bladder cancer treatment [OPTIMA] study). Preclinical results demonstratelevels of MMC in the bladder 2 hours and 6 hours following instillation were over tenfold higher as compared to drug alone (same total dosage);

- levels of MMC in the urine were higher for a longer period of time compared to drug alone;

- levels of MMC in the plasma were low: confirming safety of the treatment.

For intra-vesical chemo-therapy, hydrophobic anticancer drugs offer a distinct advantage of greater permeability through the urothelium as compared to hydrophilic drugs. This allows longer retention of therapeutic doses within the bladder tissue even after the instilled solution is washed- out after voiding. Loading the hydrophobic drugs into carriers for sustained IDD is viewed as one of the most promising strategies to ensure continuous presence of drug in the bladder with fewer or no intermittent catheterization. Muco-adhesive nanogels have been recently evaluated in porcine urinary bladder and could be promising candidate for intra-vesical delivery of hydrophobic drugs in BC therapy.

Intra-vesical instillation therapy has potential benefits including high drug concentration at the bladder and reduced systemic exposure which minimizes systemic- toxicity and side effects compared to oral medications. But repeated 
instillations are often required to extend the drug exposure, and this lowers the compliance. Indwelling intravesical devices are physical devices that can be inserted and remain in the bladder. The drug is then released from the device in a controlled and extended manner. The device can be either biodegradable or non-degradable. The former eliminates the device removal step but can provoke debris that might lead to voiding difficulties.

A reservoir-based device constructed of a completely bio-degradable elastomer supplying ciprofloxacin- $\mathrm{HCl}$ has been evaluated in vitro and showed functionality and stability. Biodegradable devices have also been studied for IDD of antimuscarinics as this could represent an alternative to oral treatment for OAB. In vitro studies in a porcine bladder- model showed effectiveness of trospium chloride ( $\mathrm{TrCl})$ when loaded onto degradable poly(lactide-co-glycolide)-based polymer carriers. Lipid-based intravesical DDSs were loaded with $\mathrm{TrCl}$ and showed a drug- release ranging from several days up to weeks.

Trials with nondegradable indwelling devices first evaluated an intravesical pump called UROS. The device was tested in Phase I/II trials.

A nonbiodegradable device, Lidocaine Releasing Intravesical System has been deemed for intravesical delivery of lidocaine. It has a double design: one tube contains solid lidocaine hydrochloride minitablets, enabling a higher dose of drug than if a solution was used, the other lumen contains nickel titanium wire, which adopts a "pretzel" conformation once inside the bladder, preventing it from being accidentally voided. It is retained in the bladder with minimal- discomfort, allowing release of lidocaine for a sustained period. A silicone container absorbs urine to dissolve the lidocaine contents, whereas the osmotic pressure created forces the solution out of the container through a small orifice in a controlled release over 14 days. Promising results were achieved when the device was tried in a rabbit- model. lidocaine concentration in the bladder tissue was $>0.1 \mu \mathrm{g} / \mathrm{g}$ during the 3 -day period of device release, while a single instillation yielded immeasurable amounts within 24 hours.

Phase I safety assessment study in healthy- volunteers. An empty device was inserted into the bladder of 7 women, while 3 women underwent cystoscopy without placement of the device. Tolerability was assessed using visual analog scales for bladder sensation and voiding behavior, which revealed that changes from baseline were similar between groups. A Phase Ib cohort study in 16 women with moderate-to-severe IC followed. Subjects received LiRIS® $200 \mathrm{mg}$ or LiRIS@ $650 \mathrm{mg}$ for 2 weeks. There was a significant improvement in bladder pain, urinary urgency, and voiding frequency.

Cystoscopic findings also improved on day 14 (day of removal) compared with day 1 . Most strikingly, of the 6 patients who were noted to have Hunner's lesion on cystoscopicinsertion, 5 demonstrated complete resolution 14 days later. Global response assessment showed an overall responder rate of $64 \%$ at day 14 and a sustained overall responder rate of $64 \% 2$ weeks later. Extended follow-up suggests that the reduction in pain was maintained for several months after the device was removed. Expanded Phase II/III clinical trials are now underway. Potentially, other therapeutic agents could be delivered by the device for the treatment of other bladder disorders.

Encapsulated DDS: There are pilot data showing the feasibility of loading an antibiotic into a non-cell-toxic microcapsule that can both penetrate bladder cells and provide slow release of the drug that retains its bactericidal properties. This system has been developed for the delivery of high doses of antibiotic to treat recalcitrant UTIs. The capsules have been prepared with co-axial electrohydrodynamic forming technology, which has shown considerable promise for the production of fibers and particles at the micro- and nanoscale. Polymethyl-silsesquioxane has showed a good biocompatibility, physiological inertness: has been inserted in the capsules together with gentamicin. In an in vitro assay, the capsules were able to kill Enterococcus 
faecalis, the most common uropathogen. The capsules were incubated with bladder epithelial cells collected from the urine of patients with UTIs. Successful delivery in bladder cells was demonstrated, but the mechanism is still unknown. High localized doses of antibiotic could be reached by introducing capsules directly into the bladder via a catheter" [46].

According Apul Goel et al., "To evaluate the impact of voiding position on uroflowmetry parameters and to assess its potential clinical implications.

We conducted a prospective study from 2013 to 2015 and included men between 18 and 77 years old who were either healthy volunteers with an International Prostate Symptom Score (IPSS) $\leq 7$ or men with benign prostate enlargement that were on alpha-blocker medication and had an IPSS 10. Participants underwent uroflowmetry and post-void residual urine (PVRU) measurements twice, once in a sitting position and once in a standing position. The participants were divided into 4 groups based on age (35 years or younger, 36 to 50 years, 51 to 60 years, and older than 60 years).

A total of 740 men with a mean age of 40.35 years were evaluated. There was no significant difference in uroflowmetry parameters until the age of 50 years between the voiding positions. In those older than 50 years, PVRU volume was significantly lower in the sitting position than the standing position, whereas voiding time was significantly higher in the sitting position than the standing position. Other uroflowmetry parameters, including maximal and average urine flow rates, were non-significant.

The voiding position plays an important role in the uroflowmetry parameters of elderly men. Voiding in the sitting position was found to be optimal for elderly men, whereas the role of the voiding position in healthy young men could not be determined" [47] (Figure 14).

According Ho-Yin Ngai et al., "Metabolic syndrome (MetS) is a cluster of metabolic abnormalities related to central adiposity / insulin resistance. Its importance is increasingly recognized as it associates with increased risks of metabolic and cardiovascular diseases. These metabolic aberrations of MetS may lead to development of benign prostatic hyperplasia (BPH) and lower urinary tract symptoms (LUTS) in men. A 26.5\%-55.6\% prevalence of MetS in men with LUTS was reported in many studies. Although the exact biological pathway is not clear yet, insulin resistance, increased visceral adiposity, sex- hormone alterations and cellular inflammatory reactions played significant roles in the related pathophysiological processes. Clinician should recognize the cardiovascular and metabolic impacts of MetS in men with LUTS, early risk- factors optimization and use of appropriate medical therapy may possibly alter or slower the progression of LUTS/BPH, and potentially avoid unnecessary morbidities and mortalities from cardiovascular and metabolic diseases for men.

MetS has been associated with a state of chronic low grade inflammation, with elevated levels of inflammation markers such as C-reactive protein (CRP) as well as

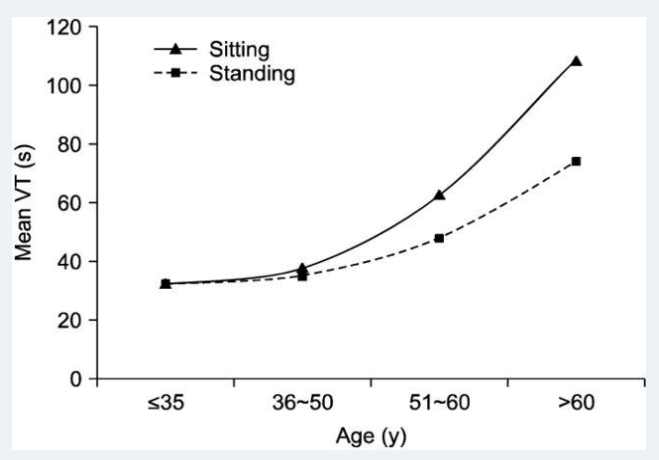

Figure 14: From refenece [47], graphic mean VT / AGE. 
pro-inflammatory cytokines such as tumor necrosis factor $\alpha$ (TNF- $\alpha$ ), interleukin (IL)-8, IL-6, and IL-1p. T-cell activities in prostate inflammatory infiltrates may result in stimulation of stromal and epithelial cell proliferation that is sustained by an autoimmune mechanism. Tissue damages and the subsequent chronic process of repetitive wound healing induced by chronic inflammation may lead to the development of BPH nodules.

Finding of inflammation in prostate biopsy in MTOPS study is associated with BPH progression. Increased serum levels of CRP have been associated with an increased risk of overall LUTS and storage LUTS.

MetS is related to arteriosclerosis. Pelvic arterial tree is not an exception. In animal model, this pelvic arteriosclerosis had been shown to cause ischaemia, resulting in thickening and fibrosis of the prostate. Neurogenic relaxation in the prostate was also impaired.

Vignozzi et al., proposed a 3-hit hypothesis on the pathogenesis of BPH under metabolic influence. An induced prostatic inflammation (1 hit) could be auto-sustained by metabolic alterations ( 2 hit) and sex steroid abnormalities ( 3 hit). This combined actions of these two or three hits, may result in overexpression of Toll-like receptors, transformation of prostatic cells into antigen-presenting cells, activation of resident human prostate-associated lymphoid tissue and over production of growth factors, therefore contributing to the prostate remodeling and enlargement" [48].

Cheng-Ling Lee et al., "The molecular etiology of BPE/BPH is complicated and poorly understood, several risk factors for the development of BPE/BPH and LUTS have been identified: age, genetics, hormones, growth factors, inflammation, lifestyle factors.

Age is the major risk factor for BPE/BPH and LUTS. The aging process involves changes in cellular mitogenesis and hormonal homeostasis in the prostate gland, which later proceed to chromosomal aberration and apoptosis. Also associated with inflammation and microvascular disease, which provoke ischemia and oxidative stress, providing a favorable environment for BPH. A genetic link for clinical BPH in men younger than 60 years has been studied over the past years. Evidence suggests that it is an inheritable disease, possibly in an autosomal dominant fashion. Moreover, the genetic factor is said to account for a $72 \%$ increased risk in developing moderate or severe LUTS in elderly men.

Sex steroid hormones have been affirmatively linked with the development and maintenance of BPE/BPH. Androgen is the most extensively studied hormone of all. In the prostate, testosterone is converted to dihydro-testosterone (DHT) by type II $5 \alpha$ reductase through DHT/androgen receptor signaling and may influence cell proliferation, differentiation, morphogenesis, and functional maintenance. The use of $5 \alpha$ reductase inhibitors in a clinical setting was found to decrease serum concentrations of DHT and slow- progression of clinical BPH. Although not yet conclusive, estrogens (both endogenous and exogenous) and selective estrogen receptor modulators may have a role in regulating stromal-epithelial interactions involved in prostatic cellular growth. There is no clear and consistent link between other sex steroid hormones and BPE/BPH.

Many growth factors and their corresponding receptors have been identified in prostatic epithelium and stroma, which can stimulate or inhibit cel-l division and differentiation processes. (Epidermal growth factor, fibroblast growth factor, and transforming growth factor- $\beta$, but this list is by no means exhaustive.) Activation of these kind of growth factors can induce stromal cell growth, followed by significant tissue remodeling, which is responsible for prostate enlargement.

Growing evidence that suggests that inflammation is closely linked to the development of BPE/BPH and LUTS. Inflammatory infiltrates are the most prevalent 
feature coexisting with $\mathrm{BPH}$, the degree of inflammation is correlated with prostate volume and weight, inflammation may activate the release of cytokines and raise the concentration of growth factors, resulting in an abnormal proliferation of prostatic cells. An increased level of C-reactive protein has been observed in men with LUTS, which is likely indicative of systemic inflammation.

In a recent systematic review of metabolic syndrome (MetS) and BPE/BPH focusing on subsets of MetS and their relationship with the TPV and LUTS, it was reported that the TPV was significantly larger in men with MetS than those without. The differences in TPV were significantly higher in obese participants and those with low serum highdensity lipoprotein cholesterol levels. Interestingly, in contrast to other studies, there were no differences in LUTS symptom scores between men with and without MetS. Heavy smoking, low physical activity, and high protein intake can also substantially alter the risks of symptomatic BPH and LUTS [49].

Bader MS et al. writed that, "UTI caused by antibiotic-resistant Gram-neg bacteria are a growing concern due to limited therapeutic options. Gram-neg, specifically Enterobacteriaceae, are common causes of community-acquired / hospital acquired UTIs. These micro- organisms can acquire genes that encode for multiple antibioticresistance mechanisms, including extended-spectrum-lactamases (ESBLs), AmpC- $\beta$ -lactamase, carbapenemases. The assessment of suspected UTI includes identification of characteristic symptoms or signs, urinalysis, dipstick or microscopic tests, and urine culture if indicated. UTIs are categorized according to the location (upper versus lower urinary tract) and severity (uncomplicated / complicated). Increasing rates of antibiotic resistance necessitate judicious use of antibiotics through the application of antimicrobial- stewardship. Knowledge of the common causative pathogens of UTIs including local susceptibility patterns are essential in determining appropriate empiric therapy. The recommended first-line empiric therapies for acute uncomplicated bacterial cystitis in otherwise healthy adult non-pregnant females is a 5-day course of nitrofurantion or a 3-g single dose of fosfomycin tromethamine. 2-line options include fluoroquinolones and $\beta$-lactams, such as amoxicillin-clavulanate. Current treatment options for the UTIs due to AmpC- $\beta$-lactamase-producing organisms include: fosfomycin, nitrofurantion, fluoroquinolones, cefepime, piperacillin-tazobactam and carbapenems. Treatment options for UTIs due to ESBLs-producing Enterobacteriaceae include nitrofurantion, fosfomycin, fluoroquinolones, cefoxitin, piperacillin-tazobactam, carbapenems, ceftazidime-avibactam, ceftolozane-tazobactam, and aminoglycosides. Based on identification and susceptibility results, alternatives to carbapenems may be used to treat mild-moderate UTIs caused by ESBL-producing Enterobacteriaceae. Ceftazidime-avibactam, colistin, polymixin B, fosfomycin, aztreonam, aminoglycosides, tigecycline are treatment options for UTIs caused by carbapenem-resistant Enterobacteriaceae (CRE). Treatment- options for UTIs caused by multidrug resistant (MDR)-Pseudomonas spp. include fluoroquinolones, ceftazidime, cefepime, piperacillintazobactam, carbapenems, aminoglycosides, colistin, ceftazidime-avibactam, and ceftolozane-tazobactam. The use of fluoro-quinolones for empiric treatment of UTIs should be restricted due to increased rates of resistance. Aminoglycosides, colistin, and tigecycline are considered alternatives in MDR Gram-negative infections in patients with limited therapeutic options" [50].

According: Huang $\mathrm{Z}$ et al., "The purpose of this study was to investigate the incidence and risk factors of male urinary tract infection (UTI) after urodynamic study (UDS). A total of 854 consecutive male patients, who underwent UDS at Peking Union Medical College Hospital from January 2010 to March 2016, were recruited in this study. Two to four weeks before the examination, urinalysis with bacterial culture was performed. Patients with negative results were selected for UDS. Immediately before the examination, urinalysis was repeated to rule out any preoperative UTI. Between 48 and $72 \mathrm{~h}$ after the exam, urine culture was performed again to determine the incidence 
of UTI. The incidence of UTI and patients' baseline characteristics, including age, medical history, urodynamic parameters, current diagnosis and pathogen type, were analyzed. Among the 854 patients undergoing UDS, urinary infection was found in 84 cases after the examination, the incidence was $9.83 \%$. Comorbidity with diabetes, post void residual (PVR), volume of prostate (Vp), and two urodynamic parameters, maximal flow rate $(\mathrm{Qmax})$ and average flow rate $(\mathrm{Qav})$ were found to be the independent risk factors for UTI after UDS. The most common pathogens were Escherichia coli (54.76\%) and Enterococcus faecalis (19.05\%). The incidence of UTI after UDS in male patients was $9.83 \%$. Patients who suffered from comorbidity of diabetes, high PVR, high Vp, low Qmax or Qav may need to be treated with prophylactic antibiotics to prevent postoperative UTI" [51].

\section{Discussion}

Lower urinary tract function is influenced by various condition like bladder overreactvity, neurological disease,Obstructions, chemical cysititis, bladder irritants substanties, infectious(upper and lower UT, complicate or not), stenosys and other with final failure of the excretory function.

Also a fine regulation, when possible, of blood glucose level in diabetes type 2, reduce recurrent cystitis.(glucose is a microbes factor growth).

But also to behavior habits, diet and other factor that can influence the complex physiology of LUT.

In some LUT disease near first choice classic drugs other molecules must be considered in order to to stop or reduce the vicious circle (BHP, chronic prostatitis).

Considering some condition like chronic prostatitis aslo under an immunologic condition can help in progression control.

An improve in pharmacokinetics is needed for example in BPH and chronic prostatitis as well as in Prostatic cancer.(better prostatic tissue perfusion of drugs).

Effective antimicrobials, right resistance profile evaluation, punctuality during of therapy,

Right associations produce better results.

(In example antimicrobial used in urinary tract infectious disease as first choice or second: trimethoprim/sulfametoxazole, nitrofurantoin, moxifloxacin, ciprofloxacin, levofloxacin,ceftriaxone, piperacillina - tazobactam, fosfomycin (oral or parenteral, meropenem, ceftazidime/avibactam,ceftolozane -tazobactam eravaciclin, plazomicyn and other).

Also new subminitration strategies can improve clinical efficacy in example top think to intra-arterious to perfuse more adequately Urotelium in interstitial cystitis. (antioxidants, anti-inflammatory molecule, antimicrobials and other).

(Innovative New PHARMACEUTICAL drug delivery systems are the right tools).

It seems the drugs cannot be not efficient because almost $95 \%$ of the drug is wasted to non-target locations even causing toxicity instead of therapeutic response. At the present time, Cyprofloxacin, antioxidants like vitamin C and vitamin E, antiinflammatory drugs, are reaching the target through urine.

In example for interstitial cyctisis If we can deliver the drugs site specifically to the capillary bed of the specific muscle, and we can sustain the drug levels at the target tissue, preventing its fall prematurely, we may be able to not only stop inflammation, and even the radical chain, causing the dysfunction of the muscles, but we even can better kill the bacteria that causes all those chains of reactions. 
Strategy must be therefore, finding a technology, which can site specifically reach those muscles without unnecessarily wasting the drug all over the body and at the same time other tissues, with not only achieving an increase of the drug level at the target, but also maintaining the drug levels for a longer period of time, stopping the drop of the concentration in the target.

This obviously, not would, but not only will increase the potency of the drug but also eliminate almost all side effects. Obviously, even with no clinical trials, we still can guess what kind of scenario we will have.

\section{The treatment of interstitial cystitis is very complex and can involve many therapeutic approaches like}

physical therapy or alternative therapies, guided imagery, massage, energy therapy, acupuncture, bladder distention, bladder instillations (mixtures including DMSO, sodium hyaluronate, Heparin, Intravesical pentosan polysulfate Intravesical chondroitin sulfate (CS), Intravesical alkalinized lidocaine, Intravesical oxybutynin and others) surgery to repair Hunner's lesions, laser surgery, neuromodulation, use of electrical nerve stimulators injections Botox.

\section{Oral medications that can be taken for the pain}

Antidepressive: Amitriptyline, histamine blokers like Cimetidine, antihistamines Hydroxyzine, Oral pentosan polysulfate, immunosoppressant like Cyclosporine A, mycophenolate, Quercetin, Hydrodistension (HD).

Triamcinolone (steroid) injection (for Hunner's lesions) narcotic - non-narcotic medications some topical medications, as lidocaine patches, vaginal or rectal diazepam, topical amitriptyline alpha blockers some antiseizure drugs like gabapentin leukotriene inhibitors, prostaglandins, as NSAIDS, ibuprofen urinary antacids, potassium or sodium citrate, tricitrates urinary tract antispasmodics, tolterodine, herbal therapies Other factor to be considered : In evolution of human species ancient monkeys progenitors was not erectus And today bath habits with sitting methods on an artificial water is different from Ancestral natural urinate process.

Also a dynamic fluxometry could be a real interesting instrument to evaluate Drugs effect (normal- basal, versus after antimicrobials, anti-inflammatory, alfa blokers Et other) in routinely urologic visits to verify on field the single patient response to drugs and to prevent vicious circle.

This make possible to reduce number of relapses when possible and a complexty reducing in Antimicrobials used preventing also resistance pattern diffusion.

According Rehan Nasir Khan et al., "Sitting position stretches the puborectalis muscle and slightly closes urogenital hiatus, causing slight obstruction at urogenital hiatus during micturition, at sitting position independent to presence or absence of any bladder outlet obstruction. This relation can also be correlated anatomically.

It was found, the average angle between rectum and anal canal was $92^{\circ}$ while sitting and becomes $132^{\circ}$ when the patient gets to squatting position. This change in angle may lead to the relaxation of puborectalis muscles leading to an easier bladder and bowel evacuation".

According the result of Rehan Nasir Khan et al. study, "In standing position the maximum flow rate was $32.4 \pm 9.6 \mathrm{ml} / \mathrm{sec}$, average flow rate $8.8 \pm 16.9 \mathrm{ml} / \mathrm{sec}$, and postvoid residue was $8.8 \pm 16.9 \mathrm{ml}$. In sitting position, the maximum flow rate was $14.8 \pm 5.4$ $\mathrm{ml} / \mathrm{sec}$, average flow rate $14.8 \pm 5.4 \mathrm{ml} / \mathrm{sec}$, and post-void residue was $6.6 \pm 9.4 \mathrm{ml}$. A significant difference in both voiding positions was observed regarding the maximum flow rate, average flow rate $(p=0.001 ; p=0.003)$. The difference was not significant 
for voided volume $(\mathrm{p}=0.676)$, or post-void residual volume $(\mathrm{p}=0.771)$ in the either position" [35]. We think then even the pee position and related change (stand or sitting in men) on urinary flow must be deeply investigated in more objective way to prevent the overuse in antimicrobials therapy if not really necessary.

A better flux, or voiding time contribute in the global bladder functions.

"The maximal flow rate (Qmax) and the average flow rate (Qav) were found to be the independent risk factors for UTI after UDS" [51].

Singh AV et al., Biohybrid cell-driven microsystems offer unparalleled possibilities for realization of soft microrobots at the micron scale. Here, we introduce a bacteriadriven microswimmer that combines the active locomotion and sensing capabilities of bacteria with the desirable encapsulation and viscoelastic properties of a soft double-micelle microemulsion for active transport and delivery of cargo (e.g., imaging agents, genes, and drugs) to living cells. Quasi-monodisperse double emulsions were synthesized with an aqueous core that encapsulated the fluorescence imaging agents, as a proof-of-concept cargo in this study, and an outer oil shell that was functionalized with streptavidin for specific and stable attachment of biotin-conjugated Escherichia coli. Motile bacteria effectively propelled the soft microswimmers across a Transwell membrane, actively delivering imaging agents (i.e., dyes) encapsulated inside of the micelles to a monolayer of cultured MCF7 breast cancer and J744A.1 macrophage cells, which enabled real-time, live-cell imaging of cell organelles, namely mitochondria, endoplasmic reticulum, and Golgi body. This in vitro model demonstrates the proofof-concept feasibility of the proposed soft microswimmers and offers promise for potential biomedical applications in active and/or targeted transport and delivery of imaging agents, drugs, stem cells, siRNA, and therapeutic genes to live tissue in in vitro disease models (e.g., organ-on-a-chip devices) and stagnant or low-flow-velocity fluidic regions of the human body" [52].

Theranostic implications of nanotechnology in multiple sclerosis: a future perspective.

Singh AV et al., "Multiple Sclerosis is a multifactorial disease with several pathogenic mechanisms and pathways. Successful MS management and medical care requires early accurate diagnosis along with specific treatment protocols based upon multifunctional nanotechnology approach. This paper highlights advances in nanotechnology that have enabled the clinician to target the brain and CNS in patient with multiple sclerosis with nanoparticles having therapeutic and imaging components. The multipartite theranostic (thera(py) + (diag)nostics) approach puts forth strong implications for medical care and cure in MS. The current nanotheranostics utilize tamed drug vehicles and contain cargo, targeting ligands, and imaging labels for delivery to specific tissues, cells, or subcellular components. A brief overview of nonsurgical nanorepair advances as future perspective is also described. Considering the potential inflammatory triggers in MS pathogenesis, a multifunctional nanotechnology approach will be needed for the prognosis" [53].

Singh AV et al., "The contamination of implant devices as a result of biofilm formation through bacterial infection has instigated major research in this area, particularly to understand the mechanism of bacterial cell/implant surface interactions and their preventions. In this paper, we demonstrate a controlled method of nanostructured titanium oxide surface synthesis using supersonic cluster beam depositions. The nanoscale surface characterization using atomic force microscopy and a profilometer display a regulated evolution in nanomorphology and physical properties. X-ray photoelectron spectroscopy analyses display a stoichiometric nanostructured TiO(2) film. Measurement of the water contact angle shows a nominal increase in the hydrophilic nature of ns-TiO(2) films, whereas the surface energy increases with decreasing contact angle. Bacterial species Staphylococcus aureus and Escherichia coli 
interaction with nanostructured surfaces shows an increase in adhesion and biofilm formation with increasing nanoscale morphological properties. Conversely, limiting ns-TiO(2) film distribution to micro/nanopatterned designed substrates integrated with bovine serum albumin functionalization leads to a reduction in biofilm formations due to a globally decreased bacterial cell-surface interaction area. The results have potential implications in inhibiting bacterial colonization and promoting mammalian cell-implant interactions" [54].

\section{Conclusion}

Related the result of bibliography analyses reported a functional - pharmacological toxicological approach can be useful In various lower urinary tract condition (global receptor profile, Bph and chronic prostatitis right therapy, bladder and prostate irritants, diabetes control, functional and anatomic strictures, neurologic disease and other).

The pathologic vicious cycle we have seen contribute in high way in progression.

New strategies, new combination of drugs, combination with fit other apic, new delivery system, biofilm agents and, micro-calcifications role in chronic prostatitis, MDR control strategies, right pharmacological p. kinetics, adeguate local drug persitance, better subministration strategies can improve the clinical endpoint.

(Observing the complete receptor equipment like an orchestra)

Normal urinary flow is a very complex - synchronized system, it depend by different factors : detrusor contractility, urotelium flogosys, urethral resistance, autonomic and voluntary nerve innervations status, abdominal straining and other factors like genetics (see some prostatic cancer) and immunologic status (see the role played in Bhp) or metabolic status (in ex diabetes, metabolic syndrome), hormonal influence, growth factors, diet (irritants), infections, water intake habit, psycological profile, stressing conditions by also to postural errors (sedentarieties, jobs), pee habit, and many other.

All factors to be considered to make possible to better manage some antimicriobials currently used in some LUT conditions.

\section{Clarifications}

This works is produced under a pharmaceutical point of view and with no any diagnostic or therapeutic intent, only to produce new research hypotesys.

\section{References}

1. Kogan $P, X$ X S, Wang Y, O'Donnell MA, Lutgendorf SK, et al. Sub-noxious Intravesical Lipopolysaccharide Triggers Bladder Inflammation and Symptom Onset in a Transgenic Autoimmune Cystitis Model: A MAPP Network Animal Study. Sci Rep. 2018; 8: 6573. Ref.: https://goo.gl/uc6w7B

2. Fiander N. Painful bladder syndrome and interstitial cystitis: treatment options. Br J Nurs. 2013; 22 : S26, S28-33. Ref.: https://goo.gl/Wue6qf

3. Wyman JF, Burgio KL, Newman DK. Practical aspects of lifestyle modifications and behavioural interventions in the treatment of overactive bladder and urgency urinary incontinence. Int $\mathrm{J}$ Clin Pract. 2009; 63: 1177-1191. Ref.: https://goo.gl/gce1LC

4. Kaddumi EG, Hubscher $\mathrm{CH}$. Urinary bladder irritation alters efficacy of vagal stimulation on rostral medullary neurons in chronic T8 spinalized rats. J Neurotrauma. 2007; 24: 1219-1228. Ref.: https://goo.gl/L2kpQZ

5. Shorter B, Lesser M, Moldwin RM, Kushner L. Effect of comestibles on symptoms of interstitial cystitis. J Urol. 2007; 178: 145-152. Ref.: https://goo.gl/zM5Gbd

6. Ellenbroek JH, Arioglu Inan E, Michel MC. A systematic review of urinary bladder hypertrophy in experimental diabetes: Part 2. Comparison of animal models and functional consequences. Neurourol Urodyn. 2018; 37: 2346-2360. Ref.: https://goo.gl/zLpvBQ 
7. Tong YC, Chin WT, Cheng JT. Role of sorbitol in the up-regulation of urinary bladder M(2) muscarinic receptors in streptozotocin-induced diabetic rats. Neurourol Urodyn. 2002; 21: 154-159. Ref.: https://goo.gl/scNz9y

8. Liu BK, Jin XW, Lu HZ, Zhang X, Zhao ZH, Shao Y. The Effects of Neurokinin-1 Receptor Antagonist in an Experimental Autoimmune Cystitis Model Resembling Bladder Pain Syndrome/Interstitial Cystitis. Inflammation. 2018; Ref.: https://goo.gl/gd9FwX

9. Butler DSC, Ambite I, Nagy K, Cafaro C, Ahmed A, et al. Neuroepithelial control of mucosal inflammation in acute cystitis. Sci Rep. 2018; 8: 11015. Ref.: https://goo.gl/pwf13G

10. Tourret J, Bagnis Cl, Denamur E. [Urinary tract infections in diabetic patients]. Rev Prat. 2014; 64: 980-983. Ref.: https://goo.gl/qWFXRy

11. Ross MA. Neuropathies associated with diabetes. Med Clin North Am. 1993; 77: 111-124. Ref.: https://goo.gl/QzNBgL

12. Hanna-Mitchell AT, Ruiz GW, Daneshgari F, Liu G, Apodaca G. Impact of diabetes mellitus on bladder uroepithelial cells. Am J Physiol Regul Integr Comp Physiol. 2013; 304: R84-R93. Ref.: https://goo.gl/26o3bQ

13. Birder L, de Groat W, Mills I, Morrison J, Thor K, et al. Neural Control of the Lower Urinary Tract: Periphera and Spinal Mechanisms. Neurourol Urodyn. 2010; 29: 128-139. Ref.: https://goo.gl/zdoDU8

14. Thor KB, Katofiasc MA, Danuser H, Springer J, Schaus JM. The role of 5-HT(1A) receptors in control of lower urinary tract function in cats. Brain Res. 2002; 946: 290-297. Ref.: https://goo.gl/DWxnss

15. luisetto M, Nili-Ahmadabadi N, Mashori GR, Sahu RK, Khan FA, et al. Immunitary role in chronic prostatitis and growth factors as promoter of BPH. Insights Clin Cell Immunol. 2018; 2: 001-013. Ref.: https://goo.gl/qfA1RL

16. Luisetto M, Nili-Ahmadabadi B. Chronic Prostatitis: The Clinical Pharmacist Role and New Delivery Systems. J Bioanal Biomed 9:e151. Ref.: https://goo.gl/dnsWrA

17. Luisetto M, Behzad NA, Ghulam RM. Relapses and Recurrent Chronic Bacteric Prostatitis - Biofilm Related, A Case Report. J of Pharmacol \& Clin Res. 2017; 4: 555644. Ref.: https://goo.gl/LkQzu8

18. Xue ZNK. Xu Z, Jia RP. [PDE5: A new therapeutic target for lower urinary tract symptoms/ benign prostatic hyperplasia combined with erectile dysfunction]. 2018; 24: 355-359. Ref.: https://goo.gl/88HQV9

19. Magri V, Marras E, Restelli A, Wagenlehner FM, Perletti G. Multimodal therapy for category III chronic prostatitis/chronic pelvic pain syndrome in UPOINTS phenotyped patients. Exp Ther Med. 2015; 9 : 658-666. Ref.: https://goo.gl/fRnfjJ

20. Ozaki T, Matsuoka J, Tsubota M, Tomita S, Sekiguchi F, et al. Zinc deficiency promotes cystitisrelated bladder pain by enhancing function and expression of $\mathrm{Ca}_{\mathrm{v}} 3.2$ in mice. Toxicology. 2018; 393 102-112. Ref.: https://goo.gl/ixt1BZ

21. Lovick TA. Central control of visceral pain and urinary tract function. Auton Neurosci. 2016; 200: 35 42. Ref.: https://goo.gl/Lc1ugW

22. Tonon L, Fromont G, Boyault S, Thomas E, Ferrari A, et al. Mutational Profile of Aggressive, Localised Prostate Cancer from African Caribbean Men Versus European Ancestry Men. Eur Urol. 2018; pii: S0302-2838(18)30618-3. Ref.: https://goo.gl/qGUQRA

23. Daneshgari F, Liu G, Birder L, Hanna-Mitchell AT, Chacko S. Diabetic Bladder Dysfunction: Current Translational Knowledge. J Urol. 2009; 182: S18-S26. Ref.: https://goo.gl/jU4zfF

24. Montalbetti N, Rued AC, Taiclet SN, Birder LA, Kullmann FA, et al. Urothelial Tight Junction Barrier Dysfunction Sensitizes Bladder Afferents. eNeuro. 2017; 4. pii: ENEURO.0381-16.2017. Ref.: https://goo.gl/HnQFRp

25. Nicholson $\mathrm{HL}$, Al-Hakeem $\mathrm{Y}$, Maldonado JJ, Tse V. Management of bladder neck stenosis and urethral stricture and stenosis following treatment for prostate cancer. Transl Androl Urol. 2017; 6(Suppl 2): S92-S102. Ref.: https://goo.gl/49P9tR

26. Zhao Z, Zhang J, He J, Zeng G. Clinical utility of the UPOINT phenotype system in Chinese males with chronic prostatitis/chronic pelvic pain syndrome (CP/CPPS): a prospective study. PLoS One. 2013; 8: e52044 Ref.: https://goo.gl/d8g6SE

27. Kim HJ, Moon H, Sohng I, Lee HW, Lee G, Lee JG. Effects of ethanol and its metabolite acetaldehyde on responses of the rat bladder. BJU Int. 1999; 83: 686-692. Ref.: https://goo.gl/VGsojr 
28. Magistro G, Wagenlehner FM, Grabe M, Weidner W, Stief CG, et al. Contemporary Management of Chronic Prostatitis/Chronic Pelvic Pain Syndrome. Eur Urol. 2016; 69: 286-297 Ref.: https://goo.gl/QHExzx

29. Bayrak O, Erturhan S, Seckiner I, Erbagci A, Ustun A, et al. Chemical cystitis developed in experimental animals model: Topical effect of intravesical ozone application to bladder. Urol Ann. 2014; 6:122126. Ref.: https://goo.gl/HJ7uiz

30. Haldar S, Dru C, Bhowmick NA. Mechanisms of hemorrhagic cystitis. Am J Clin Exp Urol. 2014; 2: 199-208. Ref.: https://goo.gl/V4F4ch

31. Golbidi S, Laher I. Bladder Dysfunction in Diabetes Mellitus. Front Pharmacol. 2010; 1: 136. Ref.: https://goo.gl/QMAUx5

32. Nitti VW. Primary Bladder Neck Obstruction in Men and Women. Rev Urol. 2005; 7(Suppl 8): S12S17. Ref.: https://goo.gl/erKX5U

33. Chen $\mathrm{C}$, Zeng $\mathrm{M}$, Xue R, Wang $\mathrm{G}$, Gao Z, et al. [Causes and management for male urethral stricture]. 2018; 43: 520-527. Ref.: https://goo.gl/q3ZRCw

34. van Rij S, Gilling P. Recent advances in treatment for Benign Prostatic Hyperplasia. Version 1. F1000Res. 2015; 4: F1000 1482. Ref.: https://goo.gl/Sg51ss

35. Khan RN, Zaidi SZ. Comparison of position-related changes on uroflowmetric parameters in healthy young men. J Pak Med Assoc. 2017; 67: 839-842. Ref.: https://goo.gl/MXijWf

36. Gravas S, Samarinas M, Zacharouli K, Karatzas A, Tzortzis V, et al. The effect of hexanic extract of Serenoa repens on prostatic inflammation: results from a randomized biopsy study. World $\mathrm{J}$ Urol. 2018; Ref.: https://goo.gl/tjHXRF

37. Morelli A, Sarchielli E, Comeglio P, Filippi S, Mancina R, et al. Phosphodiesterase type 5 expression in human and rat lower urinary tract tissues and the effect of tadalafil on prostate gland oxygenation in spontaneously hypertensive rats. J Sex Med. 2011; 8: 2746-2760. Ref.: https://goo.gl/7A6PYY

38. Fibbi B, Morelli A, Vignozzi L, Filippi S, Chavalmane A, et al. Characterization of phosphodiesterase type 5 expression and functional activity in the human male lower urinary tract. J Sex Med. 2010; 7(1 Pt 1): 59-69. Ref.: https://goo.gl/i5bhjC

39. Krivoborodov GG, Shumilo DV, Basil'ev AV, Tur El, Poddubnaia NV. [Botulinic toxin type a in combined treatment of males with chronic pelvic pain syndrome in combination with bladder emptying dysfunction]. Urologiia. 2011; 5: 67-72. Ref.: https://goo.gl/frFzff

40. Zhanel GG, Zhanel MA, Karlowsky JA. Oral Fosfomycin for the Treatment of Acute and Chronic Bacterial Prostatitis Caused by Multidrug-Resistant Escherichia coli. Can J Infect Dis Med Microbiol. 2018; 2018: 1404813. Ref.: https://goo.gl/xxC1Jx

41. Niemitz $C$. The evolution of the upright posture and gait-a review and a new synthesis. Naturwissenschaften. 2010; 97: 241-263. Ref.: https://goo.gl/Z7DTSS

42. Kwon WA. Animal Model of Interstitial Cystitis/Bladder Pain Syndrome. Int Neurourol J. 2018 Jan; 22(Suppl 1): S1-2. Ref.: https://goo.gl/W2HYwx

43. Lusty A, Kavaler E, Zakariasen K, Tolls V, Nickel JC. Treatment effectiveness in interstitial cystitis/ bladder pain syndrome: Do patient perceptions align with efficacy-based guidelines? Can Urol Assoc J. 2018; 12: E1-E5. Ref.: https://goo.gl/2kvp2u

44. Cvach K, Rosamilia A. Review of intravesical therapies for bladder pain syndrome/interstitial cystitis. Transl Androl Urol. 2015; 4: 629-637. Ref.: https://goo.gl/edK5Zi

45. Jayasimha S. Nanotechnology in Urology. Indian J Urol. 2017; 33: 13-18. Ref.: https://goo.gl/Z4YvXC

46. Zacchè MM, Srikrishna S, Cardozo L. Novel targeted bladder drug-delivery systems: a review. Res Rep Urol. 2015; 7: 169-178. Ref.: https://goo.gl/NeCvih

47. Goel A, Kanodia G, Sokhal AK, Singh K, Agrawal M. et al. Evaluation of Impact of Voiding Posture on Uroflowmetry Parameters in Men. World J Mens Health 2017; 35: 100-106. Ref.: https://goo.gl/cGRi3f

48. Ngai HY, Yuen KKS, Ng CM, Cheng CH, Chub SKP. Metabolic syndrome and benign prostatic hyperplasia: An update. Asian J Urol. 2017; 4: 164-173. Ref.: https://goo.gl/goe5c8

49. Lee CL, Kuo HC. Pathophysiology of benign prostate enlargement and lower urinary tract symptoms: Current concepts. Ci Ji Yi Xue Za Zhi. 2017; 29: 79-83. Ref.: https://goo.gl/UaeCZX

50. Bader MS, Loeb M, Brooks AA. An update on the management of urinary tract infections in the era of antimicrobial resistance. Postgrad Med. 2017; 129: 242-258. Ref.: https://goo.gl/9PpL1t 
51. Huang Z, Xiao H, Li H, Yan W, Ji Z. Analysis of the incidence and risk factors of male urinary tract infection following urodynamic study. Eur J Clin Microbiol Infect Dis. 2017; 36: 1873-1878. Ref.: https://goo.gl/FUkZkP

52. Singh AV, Hosseinidoust Z, Park BW, Yasa O, Sitti M. Microemulsion-Based Soft BacteriaDriven Microswimmers for Active Cargo Delivery. ACS Nano. 2017; 11: 9759-9769. Ref.: https://goo.gl/Ys9G62

53. Singh AV, Khare M, Gade WN, Zamboni P. Theranostic implications of nanotechnology in multiple sclerosis: a future perspective. Autoimmune Diseases. 2012; 2012, Article ID 160830: 12. Ref.: https://goo.gl/H9xkyN

54. Singh AV, Vyas V, Salve TS, Cortelli D, Dellasega D, et al. Biofilm formation on nanostructured titanium oxide surfaces and a micro/nanofabrication-based preventive strategy using colloidal lithography. Biofabrication. 2012; 4: 025001. Ref.: https://goo.gl/STBBWvd 